\title{
Microglia shape presynaptic properties at developing glutamatergic synapses
}

\author{
Bernadette Basilico $^{1}$ | Francesca Pagani ${ }^{2}$ | Alfonso Grimaldi ${ }^{2}$ | Barbara Cortese ${ }^{3}$ | \\ Silvia Di Angelantonio ${ }^{1,2}$ | Laetitia Weinhard ${ }^{4}$ | Cornelius Gross ${ }^{4}$ | Cristina Limatola,6 | \\ Laura Maggi $^{1}$ | Davide Ragozzino ${ }^{1,6}$
}

${ }^{1}$ Department of Physiology and Pharmacology, Sapienza University, Rome, Italy

${ }^{2}$ Istituto Italiano di Tecnologia, Rome, Italy ${ }^{3}$ CNR NANOTEC-Istituto di Nanotecnologia, Dept of Physics, Sapienza University, Rome, Italy

${ }^{4}$ Mouse Biology Unit, EMBL, Monterotondo, Italy

${ }^{5}$ Pasteur Institute, Department of Physiology and Pharmacology, Sapienza University, Rome, Italy

${ }^{6}$ IRCCS NEUROMED, Via Atinese, Pozzilli, Italy

Correspondence

Davide Ragozzino, Department of Physiology and Pharmacology, Sapienza University, Rome, Italy.

Email: davide.ragozzino@uniroma1.it

Funding information

European Molecular Biology Laboratory; Sapienza Università di Roma, Grant/Award Number: C26H1538M8; EMBL; Sapienza University of Rome, Grant/Award Number: C26H1538M8

\begin{abstract}
Deficient neuron-microglia signaling during brain development is associated with abnormal synaptic maturation. However, the precise impact of deficient microglia function on synaptic maturation and the mechanisms involved remain poorly defined. Here we report that mice defective in neuron-to-microglia signaling via the fractalkine receptor ( $\mathrm{C} \times 3 \mathrm{cr} 1 \mathrm{KO})$ show reduced microglial branching and altered motility and develop widespread deficits in glutamatergic neurotransmission. We characterized the functional properties of CA3-CA1 synapses in hippocampal slices from these mice and found that they display altered glutamatergic release probability, maintaining immature properties also at late developmental stages. In particular, CA1 synapses of $\mathrm{Cx} 3 \mathrm{cr} 1 \mathrm{KO}$ show (i) immature AMPA/NMDA ratio across developmental time, displaying a normal NMDA component and a defective AMPA component of EPSC; (ii) defective functional connectivity, as demonstrated by reduced current amplitudes in the input/output curve; and (iii) greater facilitation in the paired pulse ratio (PPR), suggesting decreased release probability. In addition, minimal stimulation experiments revealed that excitatory synapses have normal potency, but an increased number of failures, confirming a deficit in presynaptic release. Consistently, KO mice were characterized by higher number of silent synapses in comparison to WT. The presynaptic deficits were corrected by performing experiments in conditions of high release probability $\left(\mathrm{Ca}^{2+} / \mathrm{Mg}^{2+}\right.$ ratio 8$)$, where excitatory synapses showed normal synaptic multiplicity, AMPA/NMDA ratio, and proportion of silent synapses. These results establish that neuron-microglia interactions profoundly influence the functional maturation of excitatory presynaptic function.
\end{abstract}

\section{KEYWORDS}

CX3CR1, hippocampus, microglia, neuron-microglia interaction, synaptic development, synaptic transmission

\section{1 | INTRODUCTION}

Increasing evidence points to a relevant role of microglia in brain development and in shaping neural circuits. Microglial cells are highly dynamic, continuously extending and retracting their processes to monitor the brain parenchyma (Nimmerjahn, Kirchhoff, \& Helmchen, 2005), and directly contacting neuronal elements (Wake, Moorhouse, Jinno, Kohsaka, \& Nabekura, 2009). These contacts have been shown to depend on neuronal activity (Wake et al., 2009) and may be associated with an action of microglia on synapse formation, elimination and/or maturation. It is known that during development, microglia contribute in remodeling the synaptic circuits (Schafer et al., 2012; Tremblay, Lowery, \& Majewska, 2010) and plasticity (Parkhurst et al., 2013) and are involved in the pruning of supernumerary synapses, directly participating in the elimination of synapses by the phagocytosis of neuronal elements (Paolicelli et al., 2011; Schafer et al., 2012). In addition, it has been shown that microglia contribute to learning-induced synapse formation in the motor cortex, through brain-derived neurotrophic factor (Parkhurst et al., 2013) and promote the formation of new synapses in the developing somatosensory cortex (Miyamoto et al., 2016). 
54 LWILEY GLIA

It is also known that the failing of specific neuronal microglia signaling pathways, in particular, the deficiency of the microglial fractalkine's cognate receptor, CX3CR1, may give rise in the hippocampus to transiently reduced microglia numbers, delay in synaptic development (Paolicelli et al., 2011), decrease of functional brain connectivity, deficits in social interaction, and reduction in multisynaptic boutons (Zhan et al., 2014). In addition, deficiency of the microglial receptor CX3CR1 causes a delay in postnatal development of thalamo-cortical synapses in the barrel cortex (Hoshiko, Arnoux, Avignone, Yamamoto, \& Audinat, 2012).

Overall, these results argue that microglia play a key role specifically during brain development under physiological conditions, in addition to their known role in bridging inflammatory processes and environmental disturbances to neurodevelopmental disease (Mosser, Baptista, Arnoux, \& Audinat, 2017). It is, therefore, particularly important to define the mechanisms through which microglia affect synaptic maturation during brain development.

In this study, we analyzed the impact of neuron-microglia signaling via the CX3CL1/CX3CR1 chemokine system on glutamatergic synaptic transmission in the mouse hippocampus. We previously reported a permanent reduction of synaptic multiplicity at hippocampal synapses in Cx3cr1 knockout mice (Paolicelli et al., 2011; Zhan et al., 2014). Here we show that CA3-CA1 synapses fail to carry out key steps in synaptic maturation, maintaining immature synaptic properties until late developmental stages, and in particular showing a reduced efficiency of synaptic transmission and low AMPA/NMDA ratio. Moreover, we demonstrate that these electrophysiological deficits are the result of impaired synaptic transmission at the presynaptic level, leading to the formation of poorly reliable synapses. Our results highlight a specific requirement for microglia-neuron signaling in the functional maturation of presynaptic properties.

\section{2 | MATERIALS AND METHODS}

\subsection{Animals}

Procedures using laboratory animals were in accordance with the Italian and European guidelines and were approved by the Italian Ministry of Health in accordance with the guidelines on the ethical use of animals from the European Communities Council Directive of September 20, 2010 (2010/63/UE). All efforts were made to minimize suffering and number of animals used. For spontaneous EPSC recordings, Thy1::GFP; $\mathrm{C} \times 3 \mathrm{Cr}^{+/+}$and Thy1::GFP;Cx3 $\mathrm{Cr} 1^{\mathrm{KO} / \mathrm{KO}}$ littermates mice were used. Wild type C57BL-6J and $\mathrm{C} \times 3 \mathrm{cr} 1^{\text {GFP/GFP }}$ mice, grown by homozygous breeding, were used for immunohistochemistry and recordings of evoked synaptic currents. $C \times 3 c r 1^{+/ G F P}$ and $C \times 3 c r 1^{\text {GFP/ }}$ GFP mice were used for monitoring microglial processes motility. All experiments were performed on male mice.

\section{2 | Immunohistochemistry and confocal microscopy}

Mice were transcardially perfused with PBS and then 4\% paraformaldehyde (PFA). Whole brains were rapidly removed and post-fixed overnight in $4 \%$ PFA at $4{ }^{\circ} \mathrm{C}$ and incubated for $24 \mathrm{~h}$ in $30 \%$ sucrose solution at $4^{\circ} \mathrm{C}$. Brains were stored at $-80^{\circ} \mathrm{C}$ until sectioning. Frozen brains were cut into $40 \mu \mathrm{m}$ horizontal sections (Leica cryostat) and mounted onto glass slides. Brain sections were previously treated with a citrate buffer for antigen retrieval and then blocked (3\% goat serum in $0.3 \%$ Triton X-100) for $1 \mathrm{~h}$ at RT and incubated overnight at $4^{\circ} \mathrm{C}$ with lba1 primary antibody (1:500) in PBS plus $1 \%$ goat serum and $0.1 \%$ Triton X100; a fluorescent-labeled anti-rabbit IgG (1:250, AlexaFluor-633) was used as secondary antibody and Hoechst for nuclei visualization (1 $\mathrm{h}$ RT); after then, slices were mounted with a fluorescent mounting medium.

For morphological analysis of microglial cells, images were acquired in the CA1 stratum radiatum using an inverted confocal laser scanning microscope (FV10i Olympus) with a 40x water immersion objective and a $z$ step of $0.5 \mu \mathrm{m}$. Fixed brain slices from perfused animals were immunolabeled for lba-1 to visualize microglial cells. To obtain a comparable fluorescent signal in both genotypes, we chose not to take advantage of GFP fluorescence in $\mathrm{C} x 3 \mathrm{cr} 1 \mathrm{KO}$ mice. Consequently, we used a fluorescent-labeled secondary antibody with an emission spectrum that did not overlap with that one of GFP (AlexaFluor-633, max emission 647 nm, emission color far red).

Maximal intensity projections of lba-1 confocal images were analyzed, to obtain morphological indicators of cell complexity. Image processing was performed using ImageJ software. Maximum intensity projections of $z$-series stacks were created. Only cells whose cell body and processes were fully contained in the slice were included in the analysis. The soma area was determined by drawing a line around the cell body, by using the freehand selection tool. The extent of microglia ramification was quantified by measuring the area circumscribed by the distal ends of each process, using the polygon selection tool (arborization area) (Tremblay, Zettel, Ison, Allen, \& Majewska, 2012). For skeleton analysis, the maximum intensity projection was enhanced to visualize all microglia processes; this was followed by noise despeckling, to eliminate single-pixel background fluorescence. The resulting image was converted to a binary image and then skeletonized, using the corresponding plug-in in ImageJ. The skeletonized image was checked with the corresponding 3D stacks, to verify the physical continuity of cell processes, minimizing errors due to background signal. Total processes, endpoint and junction numbers were calculated from the skeletonized image.

\section{3 | Electrophysiology}

Acute hippocampal slices were obtained from 6- to 7-weeks-old mice, unless otherwise indicated. Animals were decapitated under halothane anesthesia. Whole brains were rapidly removed from the skull and immersed for $10 \mathrm{~min}$ in ice-cold artificial cerebrospinal fluid (ACSF), containing (in $\mathrm{mM}$ ) $\mathrm{KCl} 2.5, \mathrm{CaCl}_{2} 2.4, \mathrm{MgCl}_{2} 1.2, \mathrm{NaHPO}_{4} 1.2$, glucose $11, \mathrm{NaHCO}_{3} 26$, and glycerol 250 . The ACSF was continuously oxygenated with $95 \% \mathrm{O}_{2}$ and $5 \% \mathrm{CO}_{2}$ to maintain the physiological $\mathrm{pH}$. Horizontal $250-\mu \mathrm{m}$-thick slices were cut at $4^{\circ} \mathrm{C}$, using a Vibratome (DSK, Dosaka EM, Kyoto, Japan) and placed in a chamber filled with oxygenated ACSF containing (in $\mathrm{mM}$ ) NaCl 125, KCl 2.5, $\mathrm{CaCl}_{2} 2, \mathrm{MgCl}_{2}$ 1, $\mathrm{NaHPO}_{4}$ 1.2, $\mathrm{NaHCO}_{3} 26$, and glucose 10. Before use, slices were allowed to recover for at least $1 \mathrm{~h}$ at room temperature $\left(24 \pm 1^{\circ} \mathrm{C}\right)$. 
All recordings were performed at room temperature on slices submerged in ACSF and perfused with the same solution in the recording chamber. Spontaneous and evoked excitatory postsynaptic currents (EPSC) were recorded from CA1 pyramidal neurons at $-70 \mathrm{mV}$, using a patch clamp amplifier (Axopatch 200A, Molecular Devices). Data were filtered at $2 \mathrm{kHz}$, digitized (10 kHz), acquired using pClamp 10.0 software (Molecular Devices), and analyzed offline using Clampfit 10 (Molecular Devices). Patch pipettes (3-5 M $\Omega$ ) were filled with intracellular solution containing (in $\mathrm{mM}$ ) Cs-methanesulfonate 135, HEPES 10, MgATP 2, NaGTP 0.3, $\mathrm{CaCl}_{2} 0.4, \mathrm{MgCl}_{2}$ 2, QX-314 2, and BAPTA 5 ( $\mathrm{pH}$ adjusted to 7.3 with $\mathrm{CsOH})$. Bicuculline methochloride $(10 \mu \mathrm{M})$ was added to the extracellular solution to block $\mathrm{GABA}_{\mathrm{A}}$ receptors. For spontaneous EPSCs recordings, in order to equilibrate $\mathrm{Cl}^{-}$reversal potential at $-70 \mathrm{mV}$, we used the following $\mathrm{Cl}^{-}$-adjusted intracellular solution (in mM): Cs-methanesulfonate $135, \mathrm{CsCl} 7$, HEPES 10, MgATP 2, NaGTP 0.3, $\mathrm{MgCl}_{2}$ 2, and BAPTA 5 ( $\mathrm{pH}$ adjusted to 7.3 with $\mathrm{CsOH}$ ). Miniature EPSCs (mEPSC) were recorded after $10 \mathrm{~min}$ of bath perfusion in presence of tetrodotoxin (TTX, $1 \mu \mathrm{M})$. EPSC were evoked by electrical stimulation with theta glass tubes filled with external solution (tip 15-20 $\mu \mathrm{m}$ ). Stimulating electrodes, connected to stimulus isolation unit (Iso-stim A320, WPI), were placed in the stratum radiatum, to activate the Schaffer collaterals projecting to CA1. The position of stimulating pipettes was chosen based on fixed coordinates with respect to the recorded neuron (approximate distance $90 \mu \mathrm{m}$ toward the hippocampal sulcus and $80 \mu \mathrm{m}$ in the direction of CA2). Synaptic responses were evoked by stimulating for $100 \mu \mathrm{s}$ at $0.1 \mathrm{~Hz}$; the stimulus intensity was adjusted accordingly to the experiment. AMPA-mediated EPSC were evoked by paired pulse stimulations (interval $50 \mathrm{~ms}$ ), to determine the PPR. For input/output curves, Schaffer collaterals were stimulated at increasing intensities (0.1-10 mA). Each pulse of a given intensity was repeated 6 times, to obtain an average response. The input/output relationship of the AMPA component was determined at $-70 \mathrm{mV}$, whereas for NMDA component neurons were clamped at $+40 \mathrm{mV}$ in the presence of NBQX $(10 \mu \mathrm{M})$.

To investigate the presynaptic properties, we applied a protocol of minimal stimulation, choosing a stimulus intensity slightly stronger than that giving only failures (50 consecutive). This protocol resulted in the stimulation of one or two fibers which were discriminated during analysis, by measuring the EPSC delay. To study release probability, we manipulated the extracellular $\mathrm{Ca}^{2+}$ and $\mathrm{Mg}^{2+}$ concentrations, obtaining four different $\mathrm{Ca}^{2+} / \mathrm{Mg}^{2+}$ ratios: low $\mathrm{P}_{\mathrm{r}} \mathrm{CCa}^{2+}$ $=1 \mathrm{mM}, \mathrm{Mg}^{2+}=2 \mathrm{mM}$, ratio 0.5), physiological $P_{\mathrm{r}}\left(\mathrm{Ca}^{2+}=2 \mathrm{mM}\right.$, $\mathrm{Mg}^{2+}=1 \mathrm{mM}$, ratio 2), moderate $P_{\mathrm{r}}\left(\mathrm{Ca}^{2+}=3 \mathrm{mM}, \mathrm{Mg}^{2+}=0.6 \mathrm{mM}\right.$, ratio 5), and high $P_{\mathrm{r}}\left(\mathrm{Ca}^{2+}=4 \mathrm{mM}, \mathrm{Mg}^{2+}=0.5 \mathrm{mM}\right.$, ratio 8). Potency was defined as the mean amplitude of successful synaptic responses.

To determine the AMPA/NMDA ratio, stimulus strength was adjusted to obtain at $-70 \mathrm{mV}$ stable AMPA-mediated EPSC with an amplitude corresponding to the $50 \%$ of the maximum response. The NMDA current was recorded at $+40 \mathrm{mV}$ from the same neuron, using the same stimulus strength, after blocking the AMPA-mediated component with NBQX $(10 \mu \mathrm{M})$. In the experiments, when AMPA/NMDA ratio was evaluated in relation to release probability $\left(\mathrm{Ca}^{2+} / \mathrm{Mg}^{2+}=2\right.$ and 8), NMDA evoked currents were recorded in NBQX-free solution. In this case, NMDA peak amplitude was measured with a delay of $25 \mathrm{~ms}$ from the AMPA peak. For the evaluation of AMPA and NMDA failure rate, the stimulus intensity was set to obtain $~ 40 \%-50 \%$ of AMPA failures in $\mathrm{Ca}^{2+} / \mathrm{Mg}^{2+}=2$ and $10 \%-20 \%$ in $\mathrm{Ca}^{2+} / \mathrm{Mg}^{2+}=8$, respectively. The coefficient of variation (CV) analysis was performed as previously described (Kullmann, 1994). Briefly, AMPA and NMDA CVs were estimated for 50 consecutive trials at -70 and $+40 \mathrm{mV}$, respectively. The $\mathrm{CV}$ was calculated as the ratio of standard deviation to mean amplitude.

In an experimental set, MK-801 $(40 \mu \mathrm{M})$ was added to external solution, to assess the rate of NMDA-component blockade. During MK-801 application, stimulation was interrupted for 10 min to allow the drug to reach a stable concentration at synapses.

\subsection{Time-lapse imaging in acute hippocampal slices and analysis of microglia motility}

Acute hippocampal slices were obtained from 6- to 7-weeks-old mice as detailed above for electrophysiology and placed in oxygenated ACSF for at least $2 \mathrm{hr}$ at room temperature. Fluorescence monitoring was performed on slices perfused with ACSF at room temperature.

Time-lapse fluorescence determinations were performed using a customized digital imaging microscope. Excitation of GFP was achieved at $488 \mathrm{~nm}$, using a 1-nm-band width polychromatic light selector (Till Polychrome V), equipped with a $150 \mathrm{~W}$ xenon lamp (Till Photonics, Germany). Fluorescence was visualized using an upright microscope (Axioskope) equipped with a 40x water immersion objective (Achroplan Carl Zeiss) and a digital 12 bit CCD camera system (Sensi Cam, PCO AG, Germany). All the peripheral hardware control, image acquisition, and image processing were achieved using customized software Till Vision v. 4.0 (Till Photonics).

Microglial processes movement was monitored in the CA1 stratum radiatum by acquiring a fluorescent image every $10 \mathrm{~s}$ for $25 \mathrm{~min}$. Recorded time-lapse images of microglial process migration in brain slices were analyzed using the ImageJ "Manual Tracking" plug-in, as previously described (Pagani et al., 2015). Briefly, stacks were initially background subtracted to optimize contrast. Track positions of single processes were transferred into a new coordinate system, in which the starting position of each track was set to the origin of the coordinate system $(x=0, y=0)$. For each moving process (i), with position vector $\mathrm{Ri}[t]$, the change in position from one frame to the next $(\Delta \mathrm{Ri}$ $[t])$, and the instantaneous velocity $(v i(t))$ were given by $\Delta \mathrm{Ri}(t)=\mathrm{Ri}(t+$ $\Delta t)-\mathrm{Ri}(t)$, and $\mathrm{vi}(t)=\Delta \mathrm{Ri}(t) / \Delta t$ respectively, where $\Delta t$ is the elapsed time among the two frames. The mean velocity of each process was calculated as $\langle v\rangle=d x / d t$, expressed in $\mu \mathrm{m} / \mathrm{min}$, defining $\mathrm{dx}$ as the mean accumulated distance of each process $i$ sampled within the time interval $d t$. Migration track length and displacement (the Euclidean distance of each track) of single processes was also analyzed using the "Manual Tracking" plug-in tool.

\section{5 | Statistical analysis}

All data are presented as mean \pm SEM. Origin 6 and SigmaPlot 12 (Systat Software Inc., San Jose, CA) software were used for statistical analysis of morphological and electrophysiological data. Quantitative distributions of track parameters were analyzed with Origin 8 (OriginLab Co.) software. Statistical significances were determined 
by paired and unpaired $t$ test, one-way and two-way ANOVA, as indicated; multiple comparison procedures were performed with HolmSidak post hoc. $p$ values $<.05$ were considered significant.

\section{RESULTS}

\section{1 | Reduced microglia process branching and altered motility}

Microglia morphology was assessed in the hippocampal CA1 stratum radiatum of $\mathrm{P} 40 \mathrm{WT}$ and $\mathrm{C} \times 3 \mathrm{cr} 1 \mathrm{KO}$ mice. At this developmental stage, Cx3cr1 KO microglia display normal density and a partial recovery of functional properties (Pagani et al., 2015; Paolicelli et al., 2011). However, subtle morphological changes in branching and/or distribution may affect microglia-neuron interactions.

Immunohistochemical analysis in fixed hippocampal sections from P40 mice showed that in $\mathrm{Cx} 3 \mathrm{cr} 1 \mathrm{KO}$ mice microglia display altered morphology, while confirming normal density at this developmental stage (not shown). When the maximal intensity projection of each microglial cell was skeletonized (Figure 1a,b), morphological analysis revealed that Cx3cr1 KO microglia displayed larger soma area (Figure 1c) and reduced arborization (Figure 1d) compared to WT. Consistently, Cx3cr1 KO microglia had a higher morphological index (soma area/arborization area; Figure 1e), reflecting an increase of the cell body area with respect to the arborization area and suggesting differences in microglial basal properties (Tremblay et al., 2012). Cx3cr1 KO microglia also showed a lower number of processes (Figure 1f), endpoints, and branch points (Supporting Information Figure S1a,b) compared to WT, demonstrating that $\mathrm{C} \times 3 \mathrm{cr} 1 \mathrm{KO}$ microglia are less ramified. The analysis of single cell properties revealed the lack of a population of highly ramified microglial cells in $\mathrm{Cx} 3 \mathrm{cr} 1 \mathrm{KO}$, likely underlying the observed morphological differences (Supporting Information Figure S1e).

We also investigated the monitoring capability of microglial processes within the hippocampal parenchyma, by comparing the spontaneous motility of microglia processes in acute hippocampal slices from $\mathrm{C} \times 3 \mathrm{Cr}^{\text {+/GFP }}$ and $\mathrm{C} \times 3 \mathrm{cr} 1^{\text {GFP/GFP }}$ mice. Microglial processes were tracked by time-lapse video microscopy over 20-30 min and represented as migration paths from their point of origin (Figure $2 a, b)$. We observed that microglia from $\mathrm{C} \times 3 \mathrm{cr} 1 \mathrm{KO}$ mice showed a decrease in track length (Figure 2c) and increase in displacement (Figure 2e), but no change in the speed of process elongation (not shown). This suggests that, despite a shorter movement route, Cx3cr1 KO processes moved away from the starting point farther than their heterozygous controls, as revealed by analysis of the cumulative plots (Figure $2 d-f$ ).

Taken together, these data identify functional and morphological deficits in $\mathrm{Cx} 3 \mathrm{cr} 1 \mathrm{KO}$ microglia and argue that microglial dysfunction in these mice is not restricted to the first weeks of postnatal life.

\section{2 | Immature AMPA/NMDA ratio at CA3-CA1 hippocampal synapses}

To examine potential persistent functional defects in synaptic function associated with deficient neuron-microglia signaling, we (a)

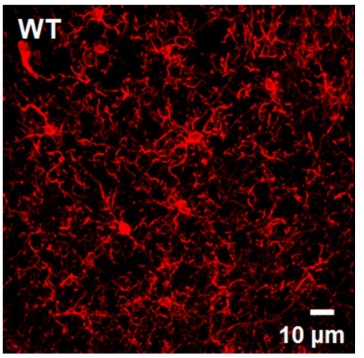

(b)

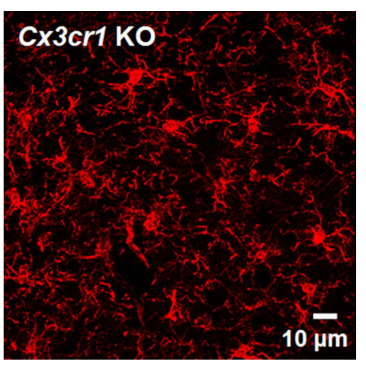

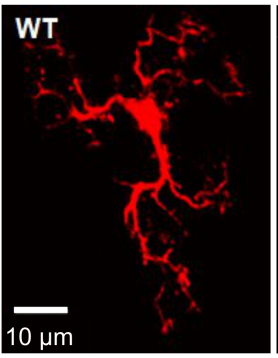

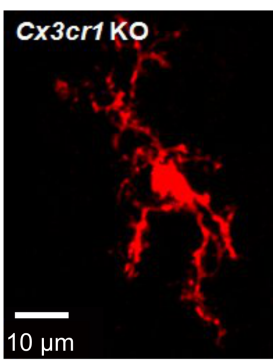

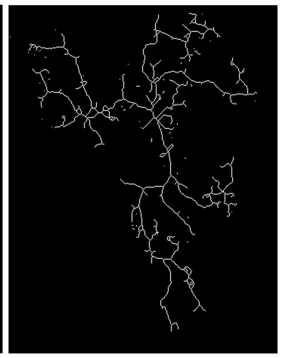

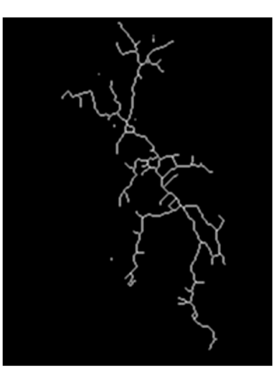

(c)

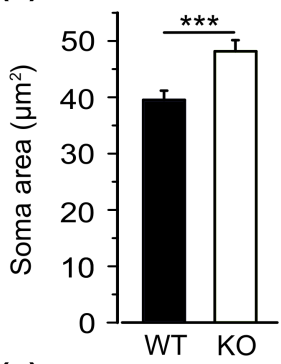

(e)

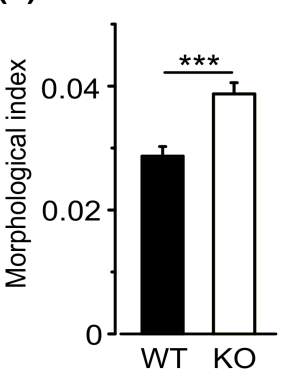

(d)

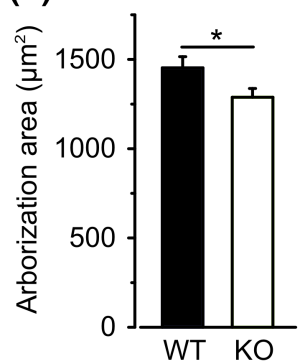

(f)

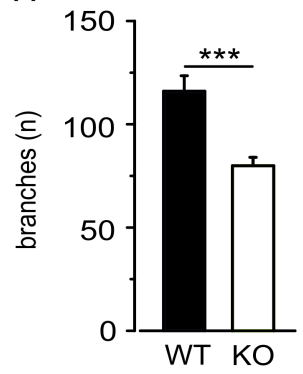

FIGURE 1 CX3cr1 KO mice show altered microglial morphology. (a) Representative maximum intensity projection (left panel) of confocal images showing Iba-1-positive microglial cells (in red) from WT hippocampal slices. Confocal image of single microglial cell and the corresponding skeleton reconstruction (right panels). (b) As in (a), maximum intensity projection (left panel) and confocal image of single microglial cell and the corresponding skeleton reconstruction (right panels) from Cx3cr1 KO hippocampal slices. (c-e) Quantitative analysis of microglial morphology in Cx3cr1 KO revealed larger soma area (WT $39.51 \pm 1.63 \mu \mathrm{m}^{2}, n=42 / 12 / 3$, cells/fields/mice, blue, vs KO $48.12 \pm 1.98 \mu \mathrm{m}^{2}, n=40 / 12 / 3$, green; Student's $t$ test; $t=3.365, * * * p<.001$ ), smaller arborization area (WT 1453.07 $\pm 62.5 \mu \mathrm{m}^{2}, n=42 / 12 / 3$, black, vs KO $1288.31 \pm 48.8 \mu \mathrm{m}^{2}, n=40 / 12 / 3$ in white; Student's $t$ test; $t=-2.063, * p=.042$ ) and higher morphological index (WT $0.028 \pm 0.0015, n=42 / 12 / 3$, black, vs KO $0.038 \pm 0.0018$, $n=40 / 12 / 3$, white; Student's $t$ test; $t=4.165$, ***p < .001), compared to WT. ( $f$ ) The number of microglia processes were significantly decreased in Cx3cr1 KO (WT $116.31 \pm 7.1, n=41 / 12 / 3$, black, vs KO $79.91 \pm 4, n=37 / 12 / 3$, white; Student's $t$ test; $t=-4.307$, ***p <.001). Scale bar $10 \mu \mathrm{m}$. Data are showed as mean \pm SEM and presented as cells/fields/mice numbers [Color figure can be viewed at wileyonlinelibrary.com] 
(a)
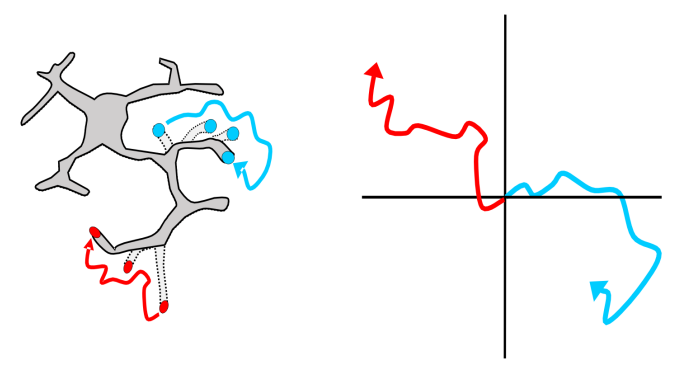

(c)

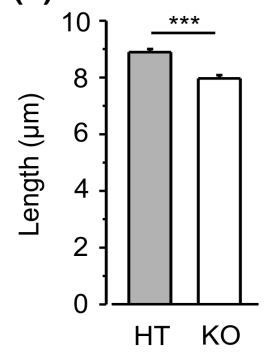

(d)

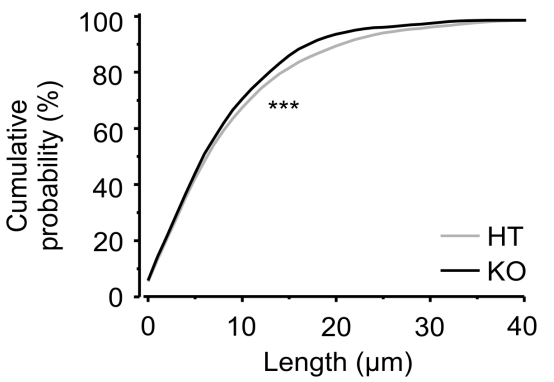

(b)
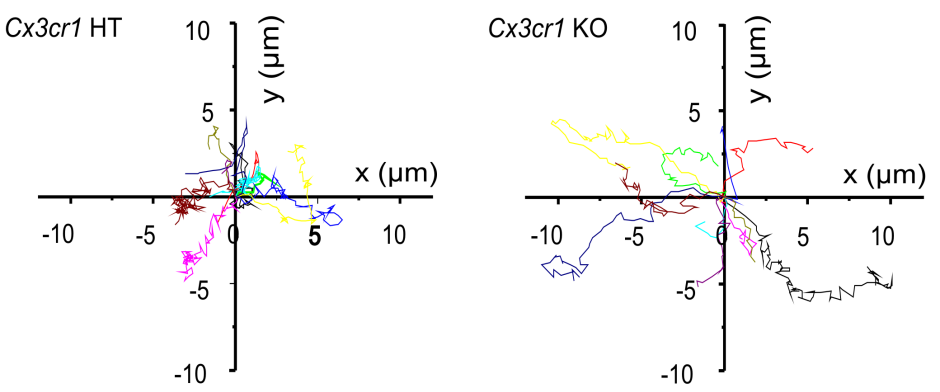

(e)

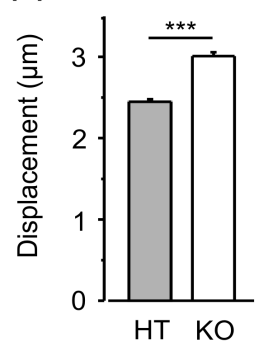

(f)

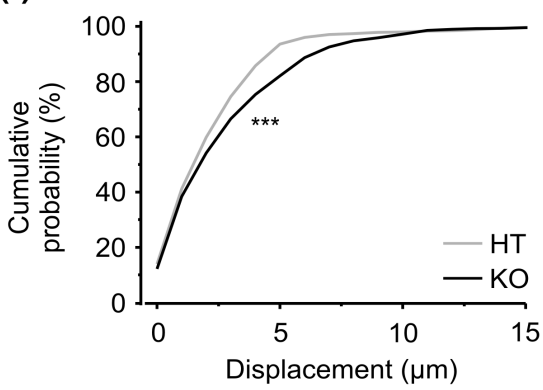

FIGURE 2 Basal microglia motility is impaired in Cx3cr1 KO. (a) Schematic representation of microglial cell, arrows represents the movement of single microglial processes. (b) Track analysis of $C \times 3 \mathrm{cr}^{+/ \mathrm{GFP}}$ (HT, left panel) and $\mathrm{C} \times 3 \mathrm{cr} 1^{\mathrm{GFP} / \mathrm{GFP}}$ (KO, right panel) microglial processes, measured by time-lapse fluorescence monitoring in acute hippocampal slices $\left(25 \mathrm{~min}\right.$ ). (c) Bar graph representing the track length of microglial processes in $\mathrm{Cx} 3 \mathrm{cr} 1^{+/ G F P}$ (grey) and $\mathrm{C} \times 3 \mathrm{cr} 1^{\mathrm{GFP} / \mathrm{GFP}}$ mice (white). $\mathrm{C} \times 3 \mathrm{cr} 1 \mathrm{KO}$ microglial processes showed a decrease in track length compared to $\mathrm{HT}$ (HT $8.85 \pm 0.11 \mu \mathrm{m}$, $n=135 / 7 / 2$, processes/fields/mice numbers, vs KO $7.93 \pm 0.12 \mu \mathrm{m}, n=88 / 6 / 3$; Student's $t$ test; $t=5.51$, $* * * p<.001$ ). (d) Cumulative probability distribution of data represented in $c(K-S$ test; $z=0.001$, ***p < .001). (e) Bar graph representing the displacement of microglial processes in $\mathrm{C} \times 3 \mathrm{cr} 1^{\mathrm{GFP} /+}$ (grey) and $\mathrm{C} \times 3 \mathrm{cr} 1^{\mathrm{GFP} / \mathrm{GFP}}$ (white). $\mathrm{C} \times 3 \mathrm{cr} 1 \mathrm{KO}$ microglial processes showed an increase in displacement compared to HT (HT $2.44 \pm 0.03 \mu \mathrm{m}, n=135 / 7 / 2$, vs KO $3 \pm 0.05 \mu \mathrm{m}, n=88 / 6 / 3$; Student's $t$ test; $t=-10.081$, *** $p<.001$ ). (f) Cumulative probability distribution of data represented in e (K-S test; $\left.z=0.002,{ }^{*} * *<.001\right)$. All distances are indicated in micrometers. Mean $\pm S E M$, processes/fields/ mice numbers [Color figure can be viewed at wileyonlinelibrary.com]

characterized postsynaptic currents evoked in CA1 pyramidal neurons by Schaffer collateral stimulation in hippocampal slices from WT and Cx3cr1 KO mice. The amplitude of AMPA receptor (AMPAR) and NMDA receptor (NMDAR)-mediated components of excitatory synaptic currents were measured at $-70 \mathrm{mV}$ and $+40 \mathrm{mV}$ of holding potential (Figure 3a), respectively, to determine AMPA/NMDA ratio (Hoshiko et al., 2012). Increased AMPA/NMDA ratio is a hallmark of mature synapses, as the proportion of postsynaptic AMPARs increases with development when compared to NMDARs (Hsia, Malenka, \& Nicoll, 1998). AMPA/NMDA ratio evaluated from the second to the fourth PNW was not significantly different between WT and Cx3cr1 KO (Figure 3c). Remarkably, AMPA/NMDA ratio increased with development in WT mice, reaching its mature value around the 6th-7th PNWs. In contrast, in Cx3cr1 KO synapses AMPA/NMDA ratio did not change significantly during development, maintaining a low, immature value into adulthood $(0.84 \pm 0.15$ in $\mathrm{KO}$, compared to $2.68 \pm 0.45$ in WT during the seventh PNW; Figure 3c). These results suggest that defective maturation in $\mathrm{C} \times 3 \mathrm{Cr} 1 \mathrm{KO}$ synapses leads to permanent dysfunction in the adult.

\subsection{Reduced input/output curve of the AMPA component}

As the maturation of the NMDA component is a crucial step in synaptic development, we tested whether adult Cx3cr1 KO mice displayed normal NMDA responses. First, we observed that the NMDA component of EPSC (recorded at $+40 \mathrm{mV}$ membrane potential in the presence of NBQX $10 \mu \mathrm{M}$ ) did not show major differences in the input/ output curve between WT and KO mice at all the ages considered (Supporting Information Figure S2a-c). Indeed, the amplitude of NMDAR-mediated responses increased similarly in the two genotypes at increasing stimulation intensity, reaching similar amplitudes. This indicated that the number/strength of functional NMDA-containing synapses is not affected by the absence of CX3CR1 signaling. It is known that developmentally controlled expression of NMDA receptor subunits leads to a shift toward a faster decay of adult NMDARmediated synaptic currents (Khazipov, Ragozzino, \& Bregestovski, 1995). We observed that this maturation process is preserved in Cx3cr1 KO mice. In fact, the decay time constants of NMDARmediated currents at CA3-CA1 synapses in KO mice were typically fast and indistinguishable from WT (Supporting Information Figure S2d,e) suggesting that NR2B-NR2A subunit substitution occurs normally during postnatal development.

On the other hand, the analysis of the AMPA component of EPSCs revealed profound differences between Cx3cr1 KO and WT mice at Schaffer collateral-CA1 synapses. Indeed, input-output curves $(\mathrm{I} / \mathrm{O})$ constructed by measuring the amplitude of AMPA currents (at $-70 \mathrm{mV}$ membrane potential), elicited by stimuli of graded intensities (Figure 4a), showed that $\mathrm{C} \times 3 \mathrm{cr} 1 \mathrm{KO}$ reached reduced amplitudes with respect to WT mice (Figure $4 b, c$ ). This result 
58 WI LEY GLIA

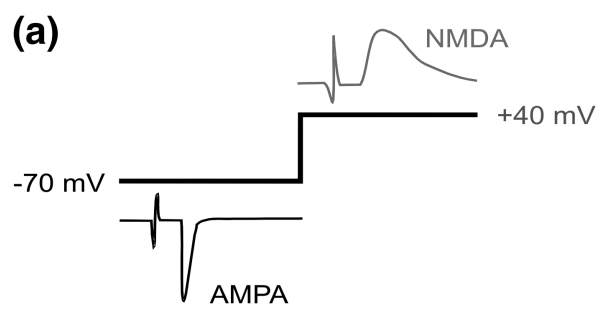

(b)

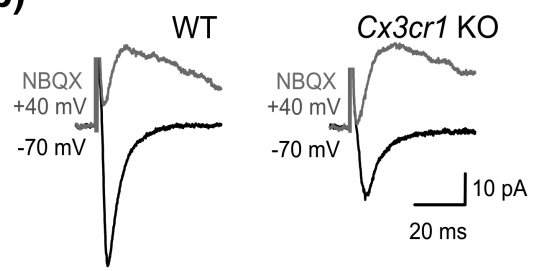

(c)

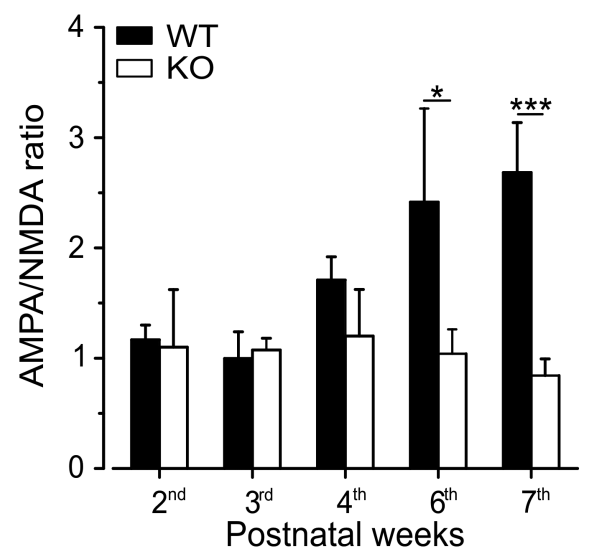

FIGURE 3 CX3cr1 KO mice display reduced AMPA/NMDA ratio at CA3-CA1 synapses. (a) Experimental design showing AMPA current evoked at $-70 \mathrm{mV}$ (black) and NMDA at $+40 \mathrm{mV}$ (grey) membrane potential. (b) Representative EPSC recorded in CA1 pyramidal cells from WT and Cx3cr1 KO hippocampal slices during the seventh PNW. Responses were evoked by Schaffer collateral stimulation as in a. (c) Bar chart illustrating the time dependent changes of AMPA/ NMDA ratio in CA1 synapses, determined measuring EPSC amplitude as in a in WT and $\mathrm{Cx} 3 \mathrm{cr} 1 \mathrm{KO}$ during postnatal development (cell number, PNW/WT/KO: 2nd/7/4; 3rd/5/11; 4th/7/4; 6th/7/6; 7th/10/17); notice the increase in ratio only in WT. Two-way ANOVA; genotype: $F(1,68)=9.094, p=.004$ age: $F(4,68)=1.719, p=.156$; interaction: $F(4,68)=2.977, p=.025$; Holm-Sidak post hoc WT (black) versus KO (white); $* p<.05, * * * p<.001$. Mean \pm SEM

suggested that in adult $\mathrm{C} \times 3 \mathrm{cr} 1 \mathrm{KO}$ mice, CA3-CA1 functional connectivity is lower, possibly due to a reduced number of functional synapses between Schaffer collaterals and CA1 pyramidal neurons or to a weakness of these synapses. Apart from the difference in amplitude, EPSCs recorded in WT and Cx3cr1 KO did not show major differences and, in particular, were characterized by similar decay kinetics ( $t=-0.564, p=.574, t$ test; not shown).

It is known that synaptic maturation during postnatal life, that leads to the reinforcement of successful synapses, is associated with an overall increase in synaptic connectivity (Hsia et al., 1998). Accordingly, when we compared I/O curves at different ages, we observed that WT mice showed a time dependent growth of peak currents, increasing roughly three times from the 3rd-4th to the 6th-7th PNWs. Remarkably, this increase was absent in Cx3cr1 KO, showing similar peak amplitudes at the two time points (Figure $4 \mathrm{~d}$ ). These findings indicate that in the absence of CX3CR1, postnatal hippocampal synapses fail in some stage of circuit maturation and refinement, leading to weaker synaptic connectivity in adulthood.

\subsection{Reduced presynaptic functionality}

To explain the observed dysfunction in glutamatergic transmission in Cx3cr1 KO mice, we analyzed excitatory synaptic currents (EPSCs) evoked in CA1 pyramidal neurons by paired stimulation of Schaffer collaterals. In this condition, we observed in $\mathrm{Cx} 3 \mathrm{cr} 1 \mathrm{KO}$ mice a higher PPR (Figure 5a,b), suggesting a presynaptic defect, associated to lower release probability. The analysis of synaptic parameters at different developmental stages revealed a time dependent decrease of PPR in WT (2nd PNW: $1.81 \pm 0.16$; 3rd-4th PNWs: $1.64 \pm 0.08$; 6th-7th PNWs: $1.4 \pm 0.06$ ), which was absent in KO (2nd PNW: $1.79 \pm 0.13$; 3rd-4th PNWs: $1.86 \pm 0.11$; 6th-7th PNWs: $1.63 \pm 0.08$ ). The presynaptic origin of the glutamatergic defect observed in the $\mathrm{KO}$ was confirmed by the analysis of mEPSCs amplitudes, which did not reveal differences between the two genotypes (WT: $5.6 \pm 0.32 \mathrm{pA}, n=17$; KO: $5.7 \pm .0 .34 \mathrm{pA}, n=14 ; t=0.274, p=.786, t$ test; not shown) (Zhan et al., 2014).

To better understand the behavior of CA3-CA1 synapses, we performed a detailed analysis of excitatory synaptic currents (EPSCs) evoked by minimal stimulation of Schaffer collaterals in CA1 pyramidal neurons (Figure 6a). Slices were equilibrated in different conditions of release probability, by changing the $\mathrm{Ca}^{2+} / \mathrm{Mg}^{2+}$ ratio of the extracellular medium, to determine changes in key functional synaptic parameters such as the number of failures and the amplitude of successes or potency (Stevens \& Wang, 1994). As expected, in physiological medium $\left(\mathrm{Ca}^{2+} / \mathrm{Mg}^{2+}\right.$ ratio 2$)$, the success rate of stimulation was low due to the relatively low release probability of CA3-CA1 synapses, with an increase observed at higher ratio. In particular, the failure rate significantly decreased in both genotypes, in parallel to the increase of $\mathrm{Ca}^{2+} / \mathrm{Mg}^{2+}$ ratio (Figure 6b, c). However, comparing the two genotypes at physiological condition, we observed that $\mathrm{Cx} 3 \mathrm{cr} 1 \mathrm{KO}$ mice showed higher number of failures $(57.8 \pm 6.5 \%)$ in respect to WT $(33.4 \pm 6.2 \%)$ and that this difference disappeared in conditions of high release probability (Figure 6b,c).

In parallel, we analyzed the potency, the average peak amplitude of successes (Stevens \& Wang, 1994). We observed that in both genotypes, potency grew in conditions of increasing release probability (Figure 6d,e), suggesting the engagement of multiple release sites (Isaac, Hjelmstad, Nicoll, \& Malenka, 1996). Remarkably, Cx3cr1 KO and WT did not show differences in potency under any of the tested conditions (Figure 6d,e), indicating that there are no relevant postsynaptic differences in the two genotypes and that the number of release sites that can be activated by the minimal stimulation protocol is similar. Altogether, these results show that $\mathrm{C} \times 3 \mathrm{cr} 1 \mathrm{KO}$ hippocampal synapses are characterized by a low release probability, indicating that synaptic defect is originated presynaptically. 
(a)
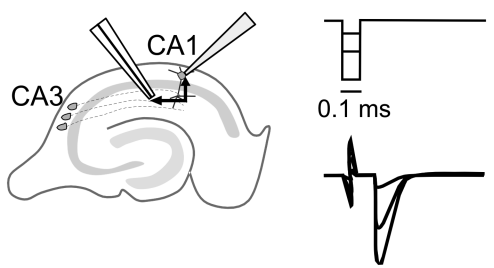

(c)

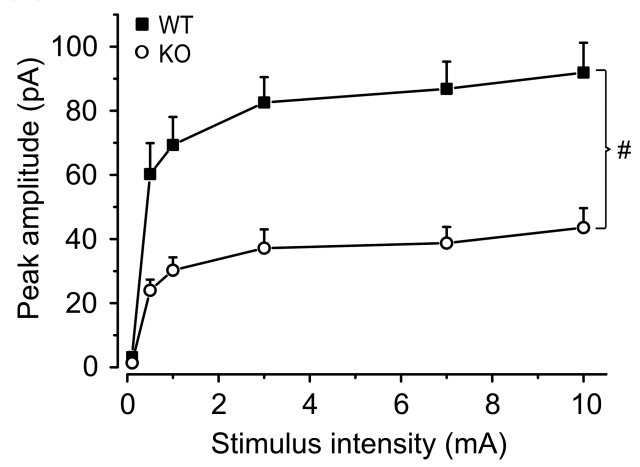

(b)
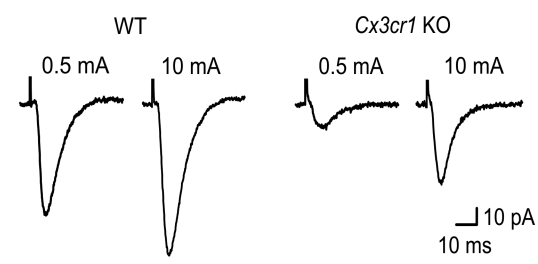

(d)

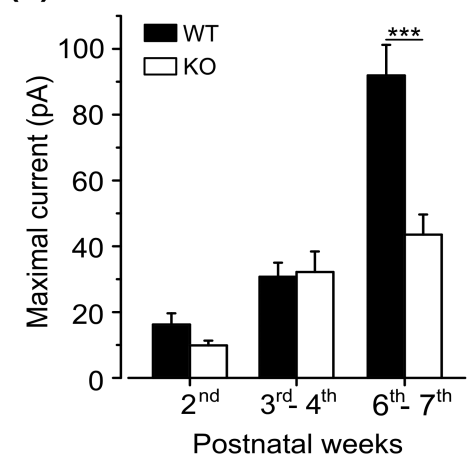

FIGURE 4 Input/output curve reveals reduced hippocampal functional connectivity in Cx3cr1 KO mice. (a) Schematic diagram showing localization of stimulating and recording electrodes in hippocampal slice. Black arrows indicate the position of electrodes (approximate distance $90 \mu \mathrm{m}$ toward the hippocampal sulcus and $80 \mu \mathrm{m}$ in the direction of CA2). On the right, schematic representation of the current steps of increasing intensity (duration $0.1 \mathrm{~ms}$ ) and the corresponding evoked responses. (b) Representative EPSCs (average of 6 traces) recorded at $-70 \mathrm{mV}$ from WT and Cx3cr1 KO neurons, following Schaffer collateral stimulation at $0.5 \mathrm{~mA}$ and $10 \mathrm{~mA}$, respectively. (c) Relationship between stimulus intensity and evoked peak amplitudes of EPSC recorded at $-70 \mathrm{mV}$ from WT $(n=21$; black squares) and $C \times 3 c r 1 \mathrm{KO}$ neurons $(n=22$; white circles). Note that Cx3cr1 KO show significantly lower peak amplitudes compared to WT. Two-way ANOvA; genotype: $F(1,246)=94.609$, $\# p<.001$; stimulus intensity: $F(5,246)=27.315, p<.001$, not indicated; interaction: $F(5,246)=3.698, p=.003$. Holm-Sidak post hoc revealed statistically significant differences at all the points considered, except for the first one, not indicated. (d) Histogram representing EPSC amplitudes evoked at the maximum stimulation intensity $(10 \mathrm{~mA})$ in WT and $\mathrm{C} \times 3 \mathrm{cr} 1 \mathrm{KO}$ during postnatal development (cell number, PNW/WT/KO: 2nd/7/5; 3rd-4th/10/11; 6th-7th/21/22). WT showed increase in amplitude with age and higher amplitudes compared to Cx3cr1 KO in the 6th-7th PNWs. Two-way ANOVA; genotype: $F(1,70)=5.273, p=.025$, not indicated; age: $F(2,70)=21.346, p<.001$, not indicated; interaction: $F$ $(2,70)=6.046, p=.004 ;$ Holm-Sidak post hoc WT, black versus KO, white, $* * * p<.001$. Mean \pm SEM

(a)

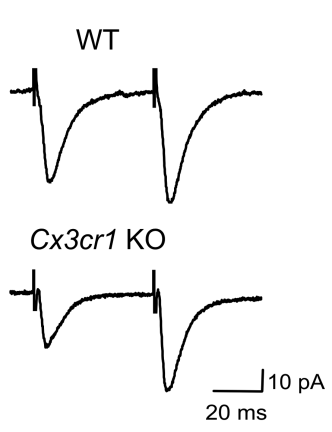

(b)

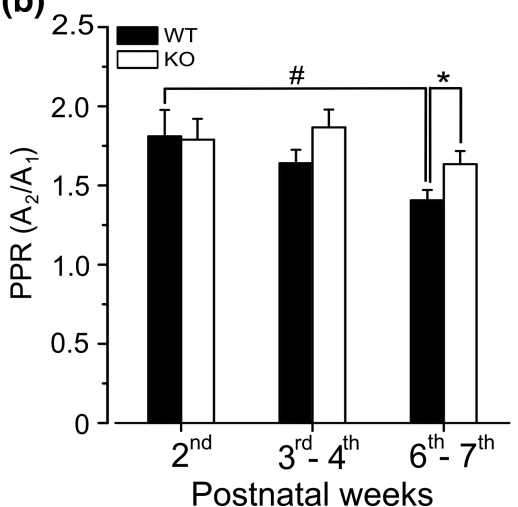

FIGURE 5 Paired pulse facilitation is higher in $\mathrm{C} \times 3 \mathrm{cr} 1 \mathrm{KO}$ synapses. (a) EPSC recorded in CA1 pyramidal cells from WT and Cx3cr1 KO hippocampal slices (6th-7th PNWs) following paired-pulse stimulation protocol (interstimulus interval 50 ms; average of 6 traces). (b) Bar graph showing the time dependent changes in PPR at WT (black) and Cx3cr1 KO (white) synapses during postnatal development (cell number, PNW/WT/KO: 2nd/8/6; 3rd-4th/15/12; 6th-7th/43/43). Note a developmental decrease of PPR only in WT. Two-way ANOVA; genotype: $F(1,121)=1.837, p=.178$; age: $F(2,121)=4.1, p=.019$, not indicated; interaction: $F(2,121)=0.452, p=.638$; Holm-Sidak post hoc, $\# p<.05, * p<.05$. Mean \pm SEM

\section{5 | Presynaptically silent synapses}

We further investigated the release probability at CA3-CA1 synapses by an independent approach based on the use-dependent block of NMDA currents by the irreversible open channel blocker MK-801 (40 $\mu$ M) (Wasling, Hanse, \& Gustafsson, 2004). Hippocampal slices from $\mathrm{C} \times 3 \mathrm{cr} 1 \mathrm{KO}$ and WT mice were treated with MK-801 to measure the progressive block rate of the NMDA component. In both genotypes, the amplitude decay of NMDA-evoked currents was fitted by a double exponential function, indicating the presence of two populations of synapses with different release probabilities. Unexpectedly, Cx3cr1 KO and WT did not show differences in decay of the NMDA response, displaying a similar blocking rate of NMDA receptors during repeated fiber stimulation (Supporting Information Figure S3). These data are only apparently in contrast with the difference in glutamate release probability estimated using AMPAmediated current recordings. Indeed, the low probability of glutamate release may not affect the activation of AMPA and NMDA receptors in the same way, due to the different affinity of the two receptors (Renger, Egles, \& Liu, 2001). The most likely explanation is that $C \times 3 c r 1$ KO mice exhibit an increased number of synapses where the release of glutamate is sufficient to activate high affinity NMDARs, but not low affinity AMPARs. 
(a)

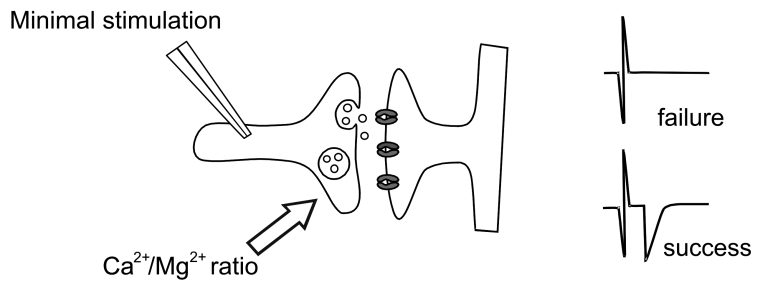

(b)

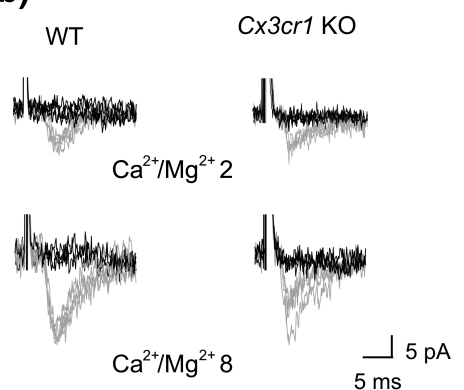

(d)

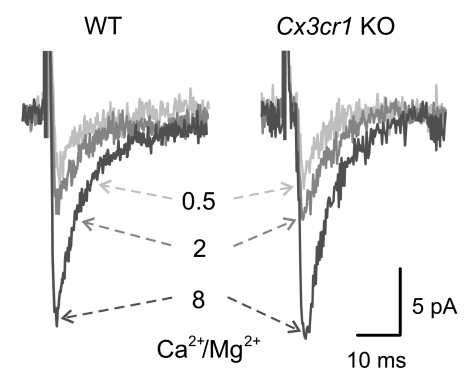

(c)

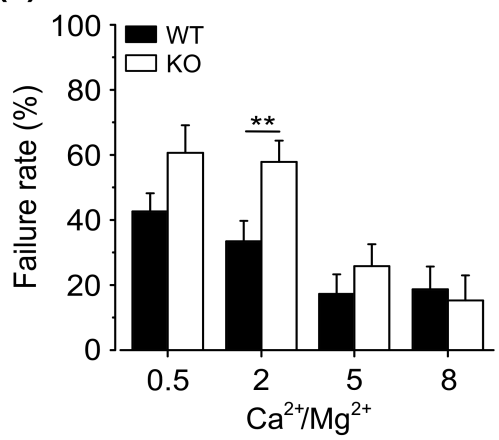

(e)

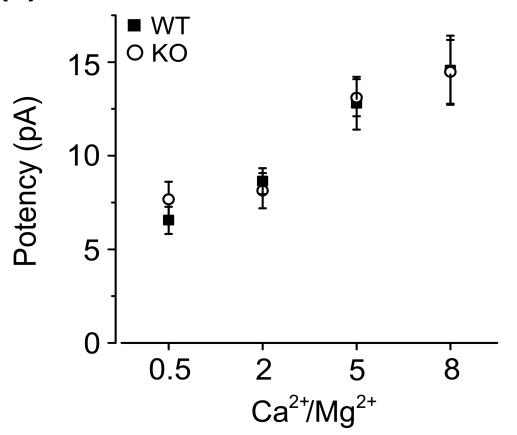

FIGURE 6 Minimal stimulation reveals synapses with lower release probability but similar potency in Cx3cr1 KO mice. (a) Experimental setup of minimal stimulation protocol performed manipulating the extracellular $\mathrm{Ca}^{2+} / \mathrm{Mg}^{2+}$ ratio. The stimulation of a single CA3 fiber is followed by a response in the postsynaptic element (success) or not (failure). (b) Superimposed representative traces recorded at $-70 \mathrm{mV}$ in WT and Cx3cr1 KO slices in $\mathrm{Ca}^{2+} / \mathrm{Mg}^{2+}$ ratio 2 and 8; note successful responses (grey) and failures (black). (c) Failure rate evaluated in different conditions of $\mathrm{Ca}^{2+} / \mathrm{Mg}^{2+}$ ratio. Note the decrease in the failure rate at increasing release probability. In physiological condition (ratio 2 ), $C x 3 \mathrm{cr} 1 \mathrm{KO}(\mathrm{n}=15$, white) exhibit a higher number of failures compared to WT ( $n=16$, black; WT: $33.4 \pm 6.2 \%$ vs KO: $57.8 \pm 6.5 \%$ ). Two-way ANovA; genotype: $F(1,73)=5.213$, $p=.025$, not indicated; $\mathrm{Ca}^{2+} / \mathrm{Mg}^{2+}$ ratio: $F(3,73)=10.638, p<.001$, not indicated; interaction: $F(3,73)=1.429, p=.241 ;$ Holm-Sidak post hoc WT versus KO; $* * p=.003$. (d) Potency (average of successful responses) of EPSC evoked at $-70 \mathrm{mV}$ in different conditions of $\mathrm{Ca}^{2+} / \mathrm{Mg}^{2+} \mathrm{ratio}$ from WT and Cx3cr1 KO pyramidal neurons. (e) Graph representing the potency of EPSC in WT (black squares) and Cx3cr1 KO (white circles), as in d. For potency evaluation, only successful responses were considered. Similar potency in conditions of increasing $\mathrm{Ca}^{2+} / \mathrm{Mg}^{2+}$ ratio suggests the presence of multiple release sites for both genotypes. Two-way ANOVA; genotype: $F(1,120)=0.061, p=.805 ; \mathrm{Ca}^{2+} / \mathrm{Mg}^{2+}$ ratio: $F(3,120)=18.489, p<.001$, not indicated; interaction: $F(3,120)=0.194, p=.9$. Mean \pm SEM

To better clarify this point and shed light onto possible differences in AMPAR and NMDAR activation rates at synapses, we measured the number of failures at $-70 \mathrm{mV}$ of membrane potential (AMPA failures) and compared it with that observed at depolarized potential (+40 mV; NMDA failures). To do this, we set the stimulation intensity to obtain a comparable failure rate in the two genotypes $(\approx 40 \%$ at $-70 \mathrm{mV})$. Results showed that in Cx3cr1 KO, NMDA failures were less pronounced than AMPA ones (AMPA failures $37.8 \pm 5.3 \%$ vs NMDA failures $22.3 \pm 3.8 \%$; Figure $7 a$,b). Conversely, in WT, the AMPA and NMDA components showed similar failure rates (AMPA failures $46.1 \pm 2.8 \%$ vs NMDA failures $39.4 \pm 2.4 \%$; Figure $7 \mathrm{a}, \mathrm{b}$ ). These data suggest that $C \times 3 \mathrm{cr} 1 \mathrm{KO}$ possess a number of AMPA-silent synapses, in which it is possible to evoke NMDA but not AMPA responses. AMPA silent synapses have been described in the immature CA1 region and disappear during postnatal development (Durand \& Konnerth, 1996). The presence of these immature synapses could explain the persistence of low AMPA/NMDA ratio in Cx3cr1 KO.

When recordings were performed in conditions of high release probability $\left(\mathrm{Ca}^{2+} / \mathrm{Mg}^{2+}\right.$ ratio 8$)$, no significant difference in failure rate was observed between AMPA and NMDA components in either genotype (Figure 7c,d), suggesting a presynaptic mechanism. Consistently, we noticed a correlation between AMPA/NMDA ratio and the number of failures in each component in all experimental groups (not shown). Notice that for $\mathrm{Ca}^{2+} / \mathrm{Mg}^{2+}$ ratio 8 experiments, the AMPA failure rate was set at $10 \%-20 \%$, in both genotypes for consistency with data illustrated in Figure 6c. The presence of presynaptically silent synapses in $\mathrm{Cx} 3 \mathrm{cr} 1 \mathrm{KO}$ was confirmed by the 
(a)
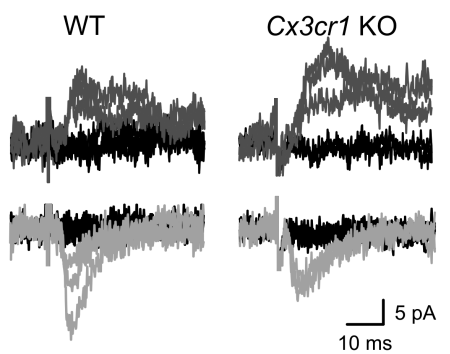

$\mathrm{Ca}^{2+} / \mathrm{Mg}^{2+} 2$

(e)

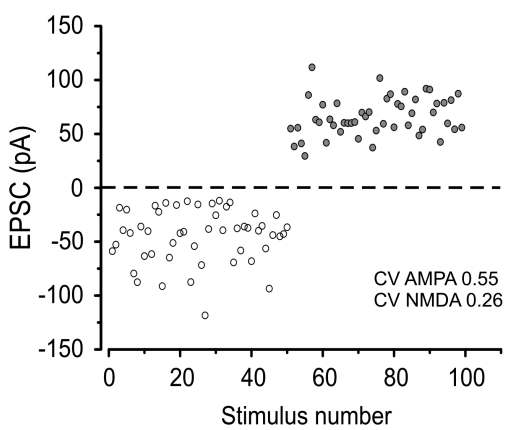

(b)

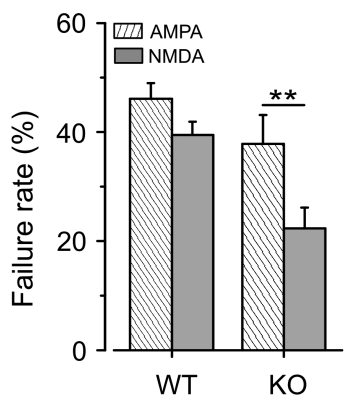

(f)

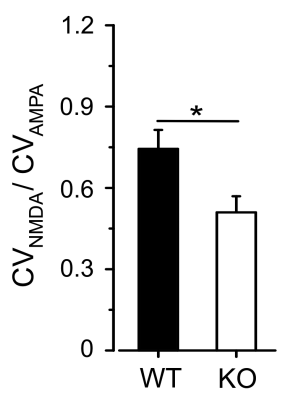

(c)
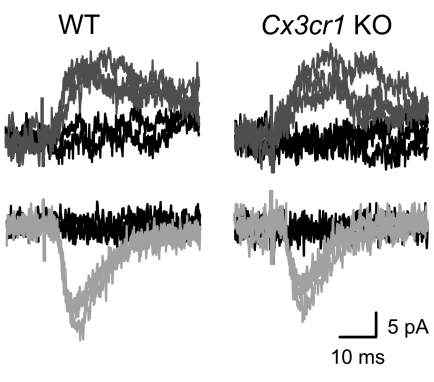

(d)

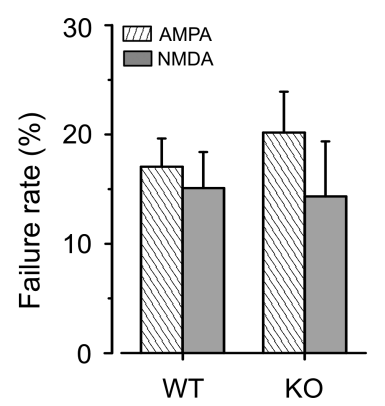

$\mathrm{Ca}^{2+} / \mathrm{Mg}^{2+} 8$

(g)

(h)

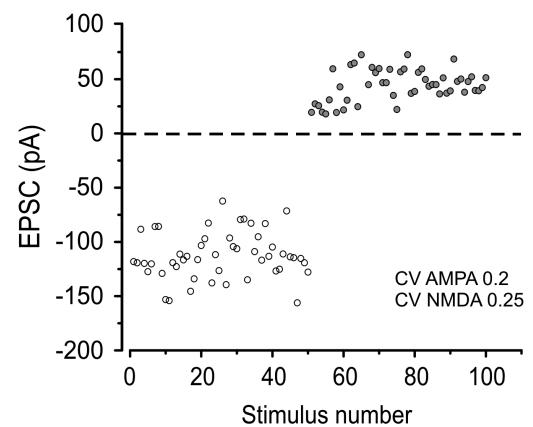

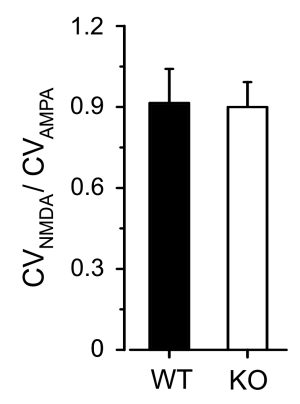

FIGURE 7 High release probability unmasks presynaptic silent synapses in Cx3cr1 KO mice. (a) Superimposed traces representing examples of failures (black) or successes (grey) recorded as in b from WT and Cx3cr1 KO hippocampal CA1 pyramidal neurons. (b) Bar chart representing the failure rate evaluated at $-70 \mathrm{mV}$ (dashed bar) and $+40 \mathrm{mV}$ (grey bar) in WT $(n=8)$ and $\mathrm{Cx} 3 \mathrm{cr} 1 \mathrm{KO}(n=9)$ in $\mathrm{Ca}^{2+} / \mathrm{Mg}^{2+}$ ratio 2 . Experiment was set to obtain for both genotypes a similar number of AMPA failures $(\approx 40 \%)$. Note the decrease of failure rate at $+40 \mathrm{mV}$, indicating the presence of AMPA-silent synapses in Cx3cr1 KO. Two-way ANOVA; genotype: $F(1,30)=10.562, p=.003$, not indicated; receptor: $F(1,30)=8.047, p=.008$, not indicated; interaction: $F(1,30)=1.285, p=.266$; Holm-Sidak post hoc AMPA versus NMDA within KO; ** $p=.007$. (c) Superimposed traces representing examples of failures (black) or successes (grey) recorded as in d from WT and Cx3cr1 KO mice. (d) Bar graph representing the failure rate evaluated in conditions of high release probability $\left(\mathrm{Ca}^{2+} / \mathrm{Mg}^{2+}\right.$ ratio 8$)$ in WT $(n=7)$ and $\mathrm{Cx} 3 \mathrm{cr} 1 \mathrm{KO}(n=11)$. Stimulation intensity was set to obtain a comparable number of AMPA failures in WT and $\mathrm{C} \times 3 \mathrm{cr} 1 \mathrm{KO}(\approx 15 \%)$. Note that in $\mathrm{Ca}^{2+} / \mathrm{Mg}^{2+}$ ratio 8 , AMPA and NMDA failure rate is similar in both genotypes. Two-way ANOVA; genotype: $F(1,32)=0.075, p=.785$; receptor: $F(1,32)=0.821, p=.372$; interaction: $F(1,32)=0.204$, $p=.655$. (e) Plot of amplitudes of AMPAR EPSCs $(-70 \mathrm{mV})$ and NMDAR EPSCs $(+40 \mathrm{mV})$ recorded in $\mathrm{Ca}^{2+} / \mathrm{Mg}^{2+}$ ratio 2 from a Cx3cr1 $\mathrm{KO}$ pyramidal neuron and their respective calculated coefficients of variation (CV). ( $f$ ) Mean CV ratios of NMDAR EPSCs to CV of AMPAR EPSCs in WT ( $n=5$; black) and Cx3cr1 KO ( $n=6$; white) neurons. Student's $t$ test; $t=-2.487$, $* p=.034$. (g) As in e, plot of amplitudes recorded in $\mathrm{Ca}^{2+} / \mathrm{Mg}^{2+}$ ratio 8. (h) Mean CV ratios of NMDAR EPSCs to CV of AMPAR EPSCs in WT ( $n=6$; black) and $\mathrm{Cx} 3 \mathrm{cr} 1 \mathrm{KO}$ ( $n=7$; white) neurons. Student's $t$ test; $t=-0.096, p=.925$. Mean \pm SEM

analysis of the coefficient of variation (CV) of the AMPA and NMDA components of EPSCs. As expected when silent and functional synapses contribute to an evoked synaptic current, in $\mathrm{KO}$, the variation of AMPAR EPSCs was larger than that of NMDAR EPSCs (Kullmann, 1994; Figure 7e), but this was not observed in WT (not shown). Consistently, the analysis of $\mathrm{CV}$ ratios (CV-NMDA/CV-AMPA) revealed that in $\mathrm{Cx} 3 \mathrm{cr} 1 \mathrm{KO}$ the $\mathrm{CV}$ ratio was lower compared to WT (Figure $7 f$ ), indicating a higher proportion of silent synapses in the KO. Remarkably, when recordings were performed in high $\mathrm{Ca}^{2+} /$ $\mathrm{Mg}^{2+}$ ratio, the $\mathrm{CV}$ ratio of EPSCs did not show differences between genotypes (Figure $7 \mathrm{~g}, \mathrm{~h}$ ). These results indicate that the higher number of failures in $\mathrm{C} \times 3 \mathrm{cr} 1 \mathrm{KO}$ is due to the presence of presynaptically silent synapses. Notably, CV analysis also indicates that the synaptic defect of $\mathrm{KO}$ is not restricted to a specific subset of low probability synapses. Altogether, these data demonstrate the presence of presynaptically silent synapses in $\mathrm{C} \times 3 \mathrm{cr} 1 \mathrm{KO}$ mice, suggesting that AMPA/NMDA ratio could be influenced by presynaptic properties.

\section{6 | Increasing release probability rescues immature synaptic properties}

To investigate this issue, we measured AMPA/NMDA ratio in EPSC recorded under high release probability conditions $\left(\mathrm{Ca}^{2+} / \mathrm{Mg}^{2+}\right.$ ratio 8). In WT, AMPA/NMDA ratio was not different compared to physiological condition $\left(\mathrm{Ca}^{2+} / \mathrm{Mg}^{2+}\right.$ ratio 8 vs 2 in WT: $t=0.948, p=.308, t$ test; not shown). Conversely, in KO, AMPA/NMDA ratio increased in $\mathrm{Ca}^{2+}$ / $\mathrm{Mg}^{2+}$ ratio 8 compared to $\mathrm{Ca}^{2+} / \mathrm{Mg}^{2+}$ ratio 2 ( $t=4.087, p<.001$, $t$ test; not shown), correcting genotypic difference (Figure $8 a, b)$. These results indicate that the AMPA/NMDA ratio is at least in part controlled by presynaptic parameters and that in $\mathrm{C} \times 3 \mathrm{cr} 1 \mathrm{KO}$, low release probability is responsible for the observed low immature AMPA/ NMDA ratio. To test whether the defect in functional connectivity of Cx3cr1 KO reported above (Figure 4) could also be dependent on low release probability, we evaluated the I/O of AMPA-mediated responses in Schaffer collaterals-CA1, in condition of high release probability $\left(\mathrm{Ca}^{2+} / \mathrm{Mg}^{2+}\right.$ ratio 8). In these conditions, we observed that PPR was similarly abolished in WT and Cx3cr1 KO mice (WT PPR: $1.08 \pm 0.09$ 
62 WILEY GLIA

vs KO PPR: $0.99 \pm 0.08 ; t=-0.756, p=.457, t$ test; data not shown), indicating a high release probability. However, the increase in release probability was not sufficient to rescue defect observed in $\mathrm{C} \times 3 \mathrm{Cr} 1 \mathrm{KO}$ mice. Indeed, Cx3cr1 KO synapses continued showing lower AMPAmediated responses at increasing stimulation, compared to WT (factor genotype: $F[1,144]=26.004, p<.001$, two-way ANOVA; data not shown), indicating that the defect in connectivity is not solely based on a reduction in release probability, but involves a reduction of efficiency or number of functional synaptic connections in $\mathrm{C} \times 3 \mathrm{cr} 1 \mathrm{KO}$.

(a)

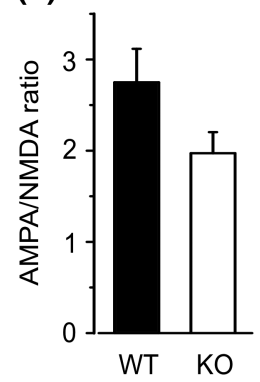

(c)

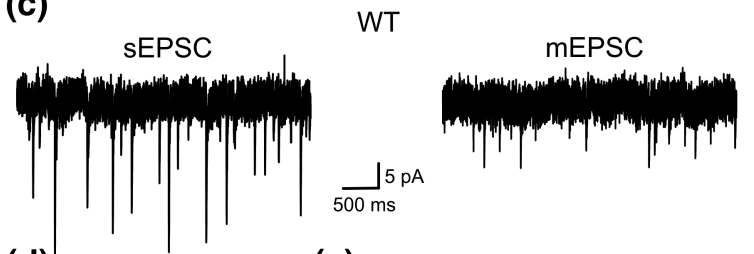

(d)

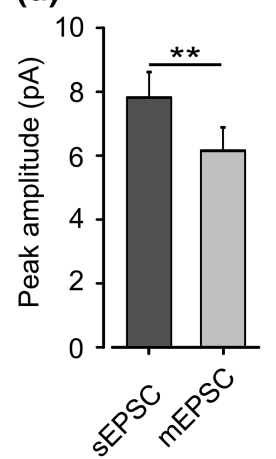

(e)

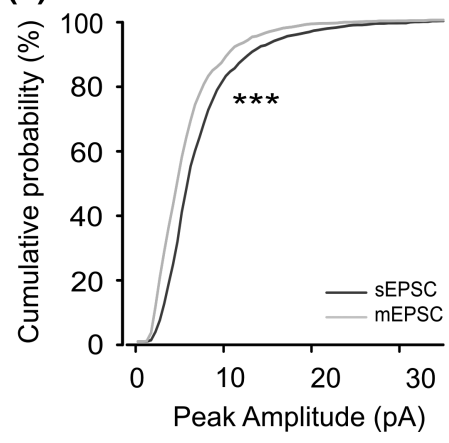

(f)

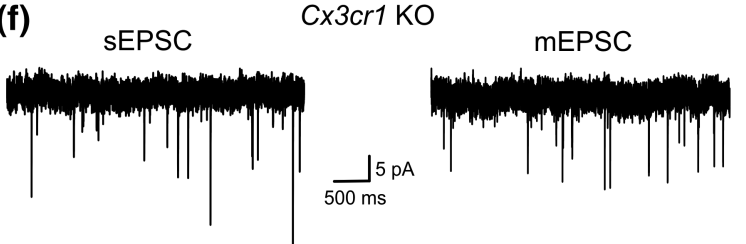

(g)

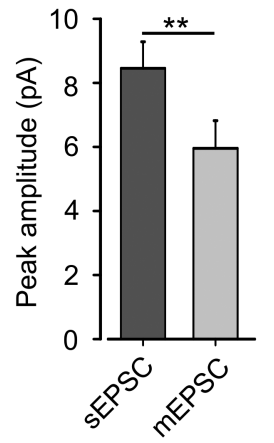

(h)

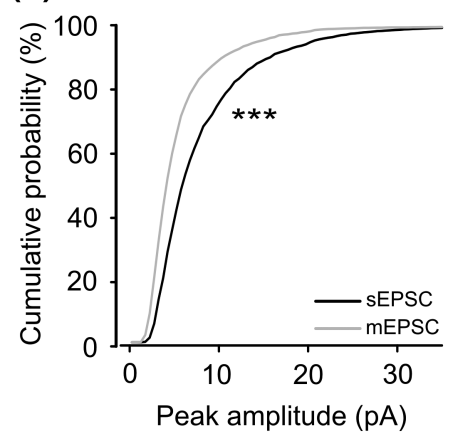

We previously reported a significant decrease in the ratio of amplitudes of spontaneous action-potential dependent (sEPSC) and miniature (mEPSC) synaptic currents in CA1 of Cx3cr1 KO mice, in the absence of differences in the amplitude or frequency of mEPSCs between the two genotypes (Zhan et al., 2014). We interpreted this defect in synaptic multiplicity as a failure in the maturation of strong synaptic contacts in KO mice (Paolicelli et al., 2011; Zhan et al., 2014). However, our present results, showing similar potency between WT and $\mathrm{Cx} 3 \mathrm{cr} 1 \mathrm{KO}$, make it unlikely that the difference in sEPSC/mEPSC amplitude ratio in the two genotypes is the result of a change in the number of synaptic release sites and we wondered whether it could be associated with the lower release probability of $\mathrm{KO}$ animals. To test this, we performed measurements of sEPSCs and mEPSCs in the two genotypes, under conditions of high release probability $\left(\mathrm{Ca}^{2+} / \mathrm{Mg}^{2+}\right.$ ratio 8). Notably, WT showed significantly larger sEPSC amplitudes compared to physiological conditions $\left(\mathrm{Ca}^{2+} / \mathrm{Mg}^{2+}\right.$ ratio 8: $7.79 \pm 0.79 \mathrm{pA}$ vs $\mathrm{Ca}^{2+} / \mathrm{Mg}^{2+}$ ratio 2: $6.22 \pm 0.49 \mathrm{pA} ; t=-3.952$, $p=.004$, paired $t$ test; not shown). A similar increase in amplitude was observed in $\mathrm{Cx} 3 \mathrm{cr} 1 \mathrm{KO}$ mice $\left(\mathrm{Ca}^{2+} / \mathrm{Mg}^{2+}\right.$ ratio 8: $8.45 \pm 0.82 \mathrm{pA}$ vs $\mathrm{Ca}^{2+} / \mathrm{Mg}^{2+}$ ratio 2: $6.26 \pm 0.47 \mathrm{pA} ; t=-3.552, p=.007$, paired $t$ test; not shown). Remarkably, under conditions of high release probability, TTX application $(1 \mu \mathrm{M})$ caused a significant decrease in current amplitudes both in WT (Figure 8c-e) and Cx3cr1 KO slices (Figure 8f-h), suggesting the presence of multiple synaptic contacts. These data show that $C \times 3 c r 1$ KO have typically multiple synaptic contacts and the defect in $\mathrm{sEPSC} / \mathrm{mEPSC}$ amplitude ratio seen in $\mathrm{KO}$ mice is a consequence of the low release probability in this genotype. Altogether, these findings demonstrate that the immature synaptic properties observed in $\mathrm{Cx} 3 \mathrm{cr} 1$ $\mathrm{KO}$ mice are largely dependent on a reduced reliability of neurotransmitter release and can be corrected increasing the release probability.

\section{4 | DISCUSSION}

Our study shows that neuron-microglia interactions profoundly influence synaptic function in the developing hippocampus. The absence

FIGURE 8 High release probability rescues AMPA/NMDA ratio and synaptic multiplicity. (a) Bar graph showing AMPA/NMDA ratio measured in condition of high release probability $\left(\mathrm{Ca}^{2+} / \mathrm{Mg}^{2+}\right.$ ratio 8$)$ in WT ( $n=15$; black) and Cx3cr1 KO ( $n=17$; white). No significant difference was detected. Student's $t$-test; $t=-1.832, p=.076$. (b) Representative traces (average of 18) recorded from WT and Cx3cr1 KO slices. Responses were evoked by Schaffer collateral stimulation at $-70 \mathrm{mV}$ (black; AMPA component) and + $40 \mathrm{mV}$ (grey; NMDA component). (c) Representative traces of sEPSCs (left) and mEPSCs (1 $\mu$ M TTX; right) from a WT CA1 pyramidal neuron in $\mathrm{Ca}^{2+}$ / $\mathrm{Mg}^{2+}$ ratio 8. (d) Bar graph of mean sEPSC (dark grey) and mEPSC (light grey) amplitude in WT in $\mathrm{Ca}^{2+} / \mathrm{Mg}^{2+}$ ratio $8(n=9)$. Student's $t$ test; $t=4.483, * * p=.004$. (e) Cumulative distributions of sEPSC (dark grey) and $\mathrm{mEPSC}$ (light grey) amplitudes as in d. K-S test; $z=0.009$, $* * * p<.001$. (f) Representative traces of sEPSCs and mEPSCs from Cx3cr1 KO as in c. (g) Bar graph of mean sEPSC (dark grey) and mEPSC (light grey) amplitude recorded in $\mathrm{C} \times 3 \mathrm{cr} 1 \mathrm{KO}$ in $\mathrm{Ca}^{2+} / \mathrm{Mg}^{2+}$ ratio 8 $(n=9)$. Student's $t$ test; $t=4.171, * * p=.003$. (h) Cumulative distributions of sEPSC (dark grey) and mEPSC (light grey) amplitudes as in $\mathrm{g}$. K-S test; $z=0.01, * * * p<.001$. Mean \pm SEM 
of CX3CR1 signaling interferes with the maturation of glutamatergic synapses and results in permanently defective synapses with reduced reliability. CA1 synapses in $\mathrm{C} \times 3 \mathrm{cr} 1 \mathrm{KO}$ animals show several immature features, failing to increase their AMPA/NMDA ratio and develop strong input/output responses. These data add to and clarify the previously reported failure to increase synaptic multiplicity (Paolicelli et al., 2011; Zhan et al., 2014). Here, we show that immature synaptic properties are the result of a profound deficit in presynaptic release probability. Reversing this deficit restores normal AMPA/NMDA and synaptic multiplicity.

It has been previously shown that neuron-microglia communication mediated by fractalkine and its receptor CX3CR1 is necessary for normal brain development. Indeed, Cx3cr1 KO show both microglia and synaptic defects (Hoshiko et al., 2012; Pagani et al., 2015; Paolicelli et al., 2011; Zhan et al., 2014), although microglia properties are reported to normalize during development (Arnoux et al., 2013; Hoshiko et al., 2012; Pagani et al., 2015; Paolicelli et al., 2011), while synaptic deficits persist into adulthood (Zhan et al., 2014). Initially, we analyzed several additional features of microglial morphology in hippocampal slices taken during the 6th-7th PNWs and found that microglia in Cx3cr1 KO mice show larger soma, reduced branching, and smaller arborization area. We also observed differences in the basal motility of microglial processes with $\mathrm{C} \times 3 \mathrm{cr} 1 \mathrm{KO}$ processes being shorter, but traveling longer distances than WT controls. We reason that this phenotype could be a consequence of the need for microglia processes to scan a larger area in $\mathrm{KO}$, due to the reduced branching in this genotype. Previous reports indicated that in the CA1 hippocampal area of $\mathrm{C} \times 3 \mathrm{cr} 1 \mathrm{KO}$ males, microglia have increased soma size and morphological index (Milior et al., 2016) along with reduced (Pagani et al., 2015) or slightly increased ramification (Milior et al., 2016). Similarly, microglia within the dentate gyrus (DG) of $\mathrm{Cx} 3 \mathrm{cr} 1 \mathrm{KO}$ animals were recently shown to have increased cell body area (Reshef, Kreisel, Beroukhim Kay, \& Yirmiya, 2014). It should be mentioned that Milior et al. (2016) used older animals (12-16 weeks) and reported larger arborization areas. We cannot exclude that discrepancies in the results are influenced by detection, as these authors used and a different approach for analyzing microglial morphology. By contrast, other authors reported no differences in microglia morphology in the DG of both adult female and male Cx3cr1 KO (Hellwig et al., 2016; Sellner et al., 2016). Notably, despite similarity in the morphologies, KO microglia showed features of activation restricted to DG area (Sellner et al., 2016), indicating that microglia function is influenced by local clues and that the correlation between morphology and functional state is not always predictable. Indeed, microglia are known to be sensitive to subtle environmental changes (Wolf, Boddeke, \& Kettenmann, 2017) and cannot be considered as a single entity, presenting varied distribution and morphology within the CNS (De Biase et al., 2017), alongside brain region-dependent differences in gene expression (Doorn et al., 2015), bioenergetics, and immunophenotype (Grabert et al., 2016). Specifically, the microenvironment in which microglia evolve influences their tissue-specific identities due to a selection pressure for exclusive gene enhancers (Gosselin et al., 2017; Lavin et al., 2014). Finally, it has to be considered that discrepancies in the literature could also arise from the age, sex or environmental conditions, including health/housing status of the animals used. Microglia react to stress stimuli (Bollinger, Bergeon Burns, \& Wellman, 2016; Tian et al., 2017) and may undergo genotype-specific changes following different types of environmental challenges (Hellwig et al., 2016; Maggi et al., 2011; Milior et al., 2016; Reshef et al., 2014; Winkler et al., 2017). Collectively, the described heterogeneities could explain some area- and context-dependent microglia properties. Nevertheless, there is a consensus that microglia function in Cx3cr1 KO is altered, displaying a reactive-like, disinhibited phenotype (Limatola \& Ransohoff, 2014). This is in line with the higher phagocytic index reported in Cx3cr1 KO microglia (Milior et al., 2016) and the lower expression of $K_{\text {ir }}$ (Pagani et al., 2015). The latter phenotype is likely to be relevant for microglia function in the unstimulated and antiinflammatory states (Lam \& Schlichter, 2015). Overall, one or more of these microglia phenotypes are likely to underlie the failure of synaptic maturation seen in $\mathrm{C} \times 3 \mathrm{cr} 1 \mathrm{KO}$ animals, possibly by interacting with environmental stimuli to influence brain function (Hellwig et al., 2016; Milior et al., 2016; Paolicelli \& Gross, 2011). However, the precise cellular and molecular mechanisms by which microglia influence synaptic maturation remain obscure (Pfeiffer, Avignone, \& Nagerl, 2016) and the remainder of our experiments were aimed at describing more precisely the synaptic deficits associated with deficient neuron-microglia signaling.

We found that hippocampal CA1 synapses in Cx3cr1 KO mice display a strong synaptic deficit, characterized primarily by a low release probability. This conclusion is based on the analysis of three parameters of synaptic function, measured varying conditions of $\mathrm{Ca}^{2+} / \mathrm{Mg}^{2+}$ ratio: PPR, failure rate, and potency. We found that at low $\mathrm{Ca}^{2+} / \mathrm{Mg}^{2+}$, the PPR was higher in $\mathrm{C} \times 3 \mathrm{cr} 1 \mathrm{KO}$ compared to WT, suggesting a lower release probability (Dobrunz \& Stevens, 1997). As expected, PPR was normalized at high $\mathrm{Ca}^{2+} / \mathrm{Mg}^{2+}$, conditions that impose high release probability. The higher PPR, together with the higher failure rate observed under conditions of minimal stimulation, strongly argues for a reduction in the probability of transmitter release in $\mathrm{KO}$ mice (Stevens \& Wang, 1994). Moreover, our results in the minimal stimulation paradigm, showing that the potency is normal in KO synapses, and the previously reported similar mEPSC amplitudes in WT and KO (Zhan et al., 2014) argue against a postsynaptic mechanism, like those reported previously (Cantaut-Belarif et al., 2017; Ragozzino et al., 2006). In addition, we found no differences in the increase in potency, typically seen at high release probability, suggesting that the number of release sites or vesicles is unchanged in the KO (Isaac et al., 1996; Stevens \& Wang, 1994). Although we cannot distinguish between the release of multiple vesicles from a single release site or the existence of multiple release sites, our interpretation is consistent with the model of multi-quantal CA1 synapses, in which a single action potential may induce the release of more than one vesicle from the same release site (Conti \& Lisman, 2003; Ricci-Tersenghi, Minneci, Sola, Cherubini, \& Maggi, 2006; Singh, Hockenberry, Tiruvadi, \& Meaney, 2011). Thus, the most likely scenario is that CA3-CA1 synapses in Cx3cr1 KO have multiple release sites operating at low probability. The absence of synaptic multiplicity observed in the Cx3cr1 KO (Paolicelli et al., 2011; Zhan et al., 2014), as evidenced by a low spontaneous/mini EPSC amplitude ratio, might thus be the consequence of inefficient release sites characterized by low probability of glutamate 
release. This interpretation is supported by the normalization of this deficit under high $\mathrm{Ca}^{2+} / \mathrm{Mg}^{2+}$ conditions.

Our data support our earlier conclusion that the synaptic phenotype seen in Cx3cr1 KO mice is due to a developmental defect. In most cases where we examined synaptic phenotypes over developmental time, KO mice showed phenotypes that emerged over time and in adulthood were indistinguishable from those of young animals. For example, CA1 synapses in Cx3cr1 KO mice show (i) decreased spontaneous/mini EPSC amplitudes, a feature that is evident from the 3rd PNW (Hsia et al., 1998); (ii) reduced AMPA/NMDA ratio, PPR, and input/output curve from the 6th PNW. Furthermore, the transient increased spine density appears at the 3rd PNW (Paolicelli et al., 2011). Indeed, AMPA/NMDA ratio is known to increase during the development of glutamatergic connections (Hsia et al., 1998), due to the delayed inclusion of AMPARs at glutamatergic postsynaptic sites (Durand, Kovalchuk, \& Konnerth, 1996) and a reduction in the AMPA component is generally interpreted as a defect in synaptic maturation (Constantine-Paton \& Cline, 1998). It is important to point out that a similar reduction in AMPA/NMDA ratio has been reported in thalamo-cortical synapses in the developing barrel cortex of $\mathrm{C} \times 3 \mathrm{cr} 1$ $\mathrm{KO}$ mice, where it was associated with a delay in the migration of microglia into the cortical barrels (Hoshiko et al., 2012). However, in our case, unlike that found in the barrel cortex, AMPA/NMDA ratio persisted into adulthood, suggesting a failure to recover this maturation deficit in the hippocampus. The decreased probability of release seen in $\mathrm{C} \times 3 \mathrm{cr} 1 \mathrm{KO}$ mice is harder to understand as a maturation deficit, as release probability is reported to decrease during hippocampal development (Wasling et al., 2004). However, this phenomenon is restricted to the 1st-2nd PNWs; and another study found that release probability increases at later developmental stages (Dumas \& Foster, 1995). Consistently with this report, our data show a developmental decrease in PPR in WT CA1 synapses, accounting for an increase in release probability. Again, this change is apparently absent in the $\mathrm{KO}$, displaying decreased probability of release at 6th-7th PNWs. In our study, the decrease in release probability was strictly dependent on the failure rate and, most importantly, limited to the AMPA component of EPSCs and thus undetectable by the MK-801 approach (Hessler, Shirke, \& Malinow, 1993) used in most studies. Somewhat unusually, in Cx3cr1 KO mice a higher number of AMPA versus NMDA failures was observed, while the two components were similar in WT animals. This could depend on the persistence in Cx3cr1 KO of silent synapses, containing only NMDARs and thus detectable only at depolarized potentials, or on the presence of presynaptically silent or inefficient synapses. Some of these, releasing a reduced amount of glutamate, only sufficient to activate high affinity NMDARs, but not AMPARs (Singh et al., 2011; Voronin \& Cherubini, 2004), possibly due to the properties of the fusion pore (Renger et al., 2001; Yoon \& Choi, 2017). Overall, our findings lead us to hypothesize the enrichment in Cx3cr1 KO mice of these pseudo-failing synapses, reported in the immature rodent CA1 (Gasparini, Saviane, Voronin, \& Cherubini, 2000; Yoon \& Choi, 2017). Notably, our findings from experiments conducted under high $\mathrm{Ca}^{2+} / \mathrm{Mg}^{2+}$ suggest that the difference in AMPA/NMDA ratio is the consequence of a deficiency in glutamate release probability. This defect could recapitulate most of the findings, which become evident in the $\mathrm{KO}$ in the same developmental window, including AMPA/NMDA ratio and a reduction in the AMPA component. We reason that this deficit may be the consequence of a reduced functionality of the $\mathrm{Ca}^{2+}$-dependent mechanisms of glutamate release, for example, $\mathrm{Ca}^{2+}$ channel properties or the sensitivity of the release machinery (Korber \& Kuner, 2016). In contrast, the properties of $\mathrm{Cx} 3 \mathrm{cr} 1 \mathrm{KO}$ could be explained by the presence of a subpopulation of postsynaptically silent synapses predominantly active at physiological conditions. In this view, the effects observed at high $\mathrm{Ca}^{2}$ ${ }^{+} / \mathrm{Mg}^{2+}$ ratio should be the consequence of the recruitment of previously inactive synapses, characterized by normal postsynaptic properties, but low release probability. The presence of population of synapses characterized by different release probability is supported by the presence of two populations in the MK801 data.

However, it should be noted that the incomplete recovery of input/output curve in $\mathrm{C} x 3 \mathrm{cr} 1 \mathrm{KO}$, under conditions of high $\mathrm{Ca}^{2+} / \mathrm{Mg}^{2+}$ ratio, argues that the reduction in AMPA component at Schaffer collaterals-CA1 synapses is not fully explainable with the decrease in release probability. This defect could rely on profound alterations in the number or properties of functional synapses both by pre- or postsynaptic mechanisms, consequently to defective neuron-microglia interaction. This suggests that in the absence of CX3CR1 signaling, CA1 synapses fail defined steps of postnatal circuitry maturation (Semple, Blomgren, Gimlin, Ferriero, \& Noble-Haeusslein, 2013), leading to permanent defects. The presence of developmental differences is further supported by the reduction in multisynaptic boutons type 1, previously reported in Cx3cr1 KO (Zhan et al., 2014). Thus, microglia dysfunction would impact also on circuit architecture in the KO, likely interfering with the structural remodeling of synaptic contacts (Weinhard et al., 2018). Indeed, microglia are known to interact dynamically with axon terminals (Tremblay et al., 2010) and they have been recently proposed to preferentially exert effects at the presynaptic level, by partial phagocytosis, or "trogocytosis" (Weinhard et al., 2018). Interestingly, depletion of microglia has been shown to alter presynaptic protein levels with known roles in synaptic function, likely affecting glutamate release (Parkhurst et al., 2013). Despite these intriguing observations, the precise role of microglia in controlling the function of the presynaptic terminals and the strengthening of the synapses remain elusive. Some factors that affect glutamatergic transmission, such as BDNF of II-1beta, TNFalpha, or ATP/adenosine (Piccinin et al., 2010; Sallert et al., 2009; Santello, Bezzi, \& Volterra, 2011), can be involved in microglia-mediated presynaptic modulation, eventually through complex intercellular loops (Pascual, Ben Achour, Rostaing, Triller, \& Bessis, 2012). These processes may be lost in the absence of fractalkine/CX3CR1 signaling, likely constituting a specific attraction clue between synapses and microglia processes and influencing the pattern of microglia-neuron contacts (Reshef et al., 2017).

Our findings demonstrate the existence of a highly specific role for neuron-microglia signaling in the maturation of glutamatergic presynaptic function during hippocampal development. We hypothesize that when such signaling is compromised, the postnatal refinement of synapses fails, leading to the formation of weaker and less functional contacts in which the reliability of glutamate release is impaired. Although we cannot entirely rule out more complicated mechanisms, including the above mentioned presence of subpopulations of 
synapses contributing preferentially to the $\mathrm{KO}$ deficit under different conditions of stimulation, we favor the interpretation that the $\mathrm{KO}$ phenotype is primarily the result of a presynaptic maturation deficit. In contrast, an intriguing way to explain our results, would be that synapses continuously need microglia interaction (by contact or soluble factors) for their correct functioning (Cantaut-Belarif et al., 2017; Parkhurst et al., 2013). Further studies will be necessary to understand the cellular and molecular mechanisms underlying the specific effect of microglia on presynaptic function and to determine whether similar processes are important in the development of other brain structures and in synaptic plasticity during normal and pathological states in adulthood (Parkhurst et al., 2013; Schafer et al., 2012). Noteworthy, the CX3CL1/CX3CR1 chemokine system is not involved in early or late forms of activity-dependent visual system plasticity (Lowery, Tremblay, Hopkins, \& Majewska, 2017; Schecter et al., 2017), indicating that fractalkine is not a universal regulator of synaptic plasticity, but has probably heterogeneous roles in specific brain regions and life stages.

\section{ACKNOWLEDGMENTS}

The work was supported by a grant from Sapienza University (C26H1538M8 to DR) and in part by funds from EMBL (to LW and CTG). BB was supported by the PhD program in Clinical-Experimental Neuroscience and Psychiatry, Sapienza University, Rome. The authors thank Francesca Zonfrillo and Alessandro Felici for animal husbandry and genotyping support. They also wish to thank Dr Piotr Bregestovski for discussion and critical revision of the manuscript and Drs Marie Eve Tremblay, Lorenzo Cingolani, and Etienne Audinat for helpful discussions.

\section{CONFLICT OF INTEREST}

The authors declare that they have no conflict of interest.

\section{AUTHOR CONTRIBUTIONS}

BB carried out and analyzed all evoked EPSC and dynamic fluorescence imaging experiments. FP performed and analyzed SEPSC experiments. $\mathrm{BC}$ performed tracking analysis. AG performed immunohistochemistry, confocal acquisition, and morphology analysis with support from BB and LW. BB and DR designed the experiments with input from LM and SDA. DR and BB wrote the article with the help of LM, CTG, and $\mathrm{CL}$. DR conceived the project.

\section{ORCID}

Davide Ragozzino (1D) https://orcid.org/0000-0002-3290-7665

\section{REFERENCES}

Arnoux, I., Hoshiko, M., Mandavy, L., Avignone, E., Yamamoto, N., \& Audinat, E. (2013). Adaptive phenotype of microglial cells during the normal postnatal development of the somatosensory "barrel" cortex. Glia, 61(10), 1582-1594. https://doi.org/10.1002/glia.22503

Bollinger, J. L., Bergeon Burns, C. M., \& Wellman, C. L. (2016). Differential effects of stress on microglial cell activation in male and female medial prefrontal cortex. Brain, Behavior, and Immunity, 52, 88-97. https://doi. org/10.1016/j.bbi.2015.10.003

Cantaut-Belarif, Y., Antri, M., Pizzarelli, R., Colasse, S., Vaccari, I., Soares, S., ... Bessis, A. (2017). Microglia control the glycinergic but not the GABAergic synapses via prostaglandin E2 in the spinal cord. The Journal of Cell Biology, 216(9), 2979-2989. https://doi.org/10.1083/ jcb.201607048

Constantine-Paton, M., \& Cline, H. T. (1998). LTP and activity-dependent synaptogenesis: The more alike they are, the more different they become. Current Opinion in Neurobiology, 8(1), 139-148.

Conti, R., \& Lisman, J. (2003). The high variance of AMPA receptor- and NMDA receptor-mediated responses at single hippocampal synapses: Evidence for multiquantal release. Proceedings of the National Academy of Sciences of the United States of America, 100(8), 4885-4890. https:// doi.org/10.1073/pnas.0630290100

De Biase, L. M., Schuebel, K. E., Fusfeld, Z. H., Jair, K., Hawes, I. A., Cimbro, R., ... Bonci, A. (2017). Local cues establish and maintain region-specific phenotypes of basal ganglia microglia. Neuron, 95(2), 341-356 e346. https://doi.org/10.1016/j.neuron.2017.06.020

Dobrunz, L. E., \& Stevens, C. F. (1997). Heterogeneity of release probability, facilitation, and depletion at central synapses. Neuron, 18(6), 995-1008.

Doorn, K. J., Breve, J. J., Drukarch, B., Boddeke, H. W., Huitinga, I., Lucassen, P. J., \& van Dam, A. M. (2015). Brain region-specific gene expression profiles in freshly isolated rat microglia. Frontiers in Cellular Neuroscience, 9, 84. https://doi.org/10.3389/fncel.2015.00084

Dumas, T. C., \& Foster, T. C. (1995). Developmental increase in CA3-CA1 presynaptic function in the hippocampal slice. Journal of Neurophysiology, 73(5), 1821-1828. https://doi.org/10.1152/jn.1995.73.5.1821

Durand, G. M., \& Konnerth, A. (1996). Long-term potentiation as a mechanism of functional synapse induction in the developing hippocampus. Journal of Physiology, Paris, 90(5-6), 313-315.

Durand, G. M., Kovalchuk, Y., \& Konnerth, A. (1996). Long-term potentiation and functional synapse induction in developing hippocampus. Nature, 381(6577), 71-75. https://doi.org/10.1038/381071a0

Gasparini, S., Saviane, C., Voronin, L. L., \& Cherubini, E. (2000). Silent synapses in the developing hippocampus: Lack of functional AMPA receptors or low probability of glutamate release? Proceedings of the National Academy of Sciences of the United States of America, 97(17), 9741-9746. https://doi.org/10.1073/pnas.170032297

Gosselin, D., Skola, D., Coufal, N. G., Holtman, I. R., Schlachetzki, J. C. M., Sajti, E., ... Glass, C. K. (2017). An environment-dependent transcriptional network specifies human microglia identity. Science, 356(6344), eaal3222. https://doi.org/10.1126/science.aal3222

Grabert, K., Michoel, T., Karavolos, M. H., Clohisey, S., Baillie, J. K., Stevens, M. P., ... McColl, B. W. (2016). Microglial brain region-dependent diversity and selective regional sensitivities to aging. Nature Neuroscience, 19(3), 504-516. https://doi.org/10.1038/nn. 4222

Hellwig, S., Brioschi, S., Dieni, S., Frings, L., Masuch, A., Blank, T., \& Biber, K. (2016). Altered microglia morphology and higher resilience to stress-induced depression-like behavior in CX3CR1-deficient mice. Brain, Behavior, and Immunity, 55, 126-137. https://doi.org/10.1016/j. bbi.2015.11.008

Hessler, N. A., Shirke, A. M., \& Malinow, R. (1993). The probability of transmitter release at a mammalian central synapse. Nature, 366(6455), 569-572. https://doi.org/10.1038/366569a0

Hoshiko, M., Arnoux, I., Avignone, E., Yamamoto, N., \& Audinat, E. (2012). Deficiency of the microglial receptor CX3CR1 impairs postnatal functional development of thalamocortical synapses in the barrel cortex. The Journal of Neuroscience, 32(43), 15106-15111. https://doi.org/10. 1523/JNEUROSCI.1167-12.2012

Hsia, A. Y., Malenka, R. C., \& Nicoll, R. A. (1998). Development of excitatory circuitry in the hippocampus. Journal of Neurophysiology, 79(4), 2013-2024. https://doi.org/10.1152/jn.1998.79.4.2013

Isaac, J. T., Hjelmstad, G. O., Nicoll, R. A., \& Malenka, R. C. (1996). Long-term potentiation at single fiber inputs to hippocampal CA1 pyramidal cells. Proceedings of the National Academy of Sciences of the United States of America, 93(16), 8710-8715.

Khazipov, R., Ragozzino, D., \& Bregestovski, P. (1995). Kinetics and Mg2+ block of $\mathrm{N}$-methyl-D-aspartate receptor channels during postnatal 
development of hippocampal CA3 pyramidal neurons. Neuroscience, 69(4), 1057-1065.

Korber, C., \& Kuner, T. (2016). Molecular machines regulating the release probability of synaptic vesicles at the active zone. Frontiers in Synaptic Neuroscience, 8, 5. https://doi.org/10.3389/fnsyn.2016.00005

Kullmann, D. M. (1994). Amplitude fluctuations of dual-component EPSCs in hippocampal pyramidal cells: Implications for long-term potentiation. Neuron, 12(5), 1111-1120.

Lam, D., \& Schlichter, L. C. (2015). Expression and contributions of the Kir2.1 inward-rectifier $\mathrm{K}(+)$ channel to proliferation, migration and chemotaxis of microglia in unstimulated and anti-inflammatory states. Frontiers in Cellular Neuroscience, 9, 185. https://doi.org/10.3389/ fncel.2015.00185

Lavin, Y., Winter, D., Blecher-Gonen, R., David, E., Keren-Shaul, H., Merad, M., ... Amit, I. (2014). Tissue-resident macrophage enhancer landscapes are shaped by the local microenvironment. Cell, 159(6), 1312-1326. https://doi.org/10.1016/j.cell.2014.11.018

Limatola, C., \& Ransohoff, R. M. (2014). Modulating neurotoxicity through CX3CL1/CX3CR1 signaling. Frontiers in Cellular Neuroscience, 8, 229. https://doi.org/10.3389/fncel.2014.00229

Lowery, R. L., Tremblay, M. E., Hopkins, B. E., \& Majewska, A. K. (2017). The microglial fractalkine receptor is not required for activity-dependent plasticity in the mouse visual system. Glia, 65(11), 1744-1761. https://doi.org/10.1002/glia.23192

Maggi, L., Scianni, M., Branchi, I., D'Andrea, I., Lauro, C., \& Limatola, C. (2011). CX(3)CR1 deficiency alters hippocampal-dependent plasticity phenomena blunting the effects of enriched environment. Frontiers in Cellular Neuroscience, 5, 22. https://doi.org/10.3389/fncel.2011.00022

Milior, G., Lecours, C., Samson, L., Bisht, K., Poggini, S., Pagani, F., ... Maggi, L. (2016). Fractalkine receptor deficiency impairs microglial and neuronal responsiveness to chronic stress. Brain, Behavior, and Immunity, 55, 114-125. https://doi.org/10.1016/j.bbi.2015.07.024

Miyamoto, A., Wake, H., Ishikawa, A. W., Eto, K., Shibata, K., Murakoshi, H., ... Nabekura, J. (2016). Microglia contact induces synapse formation in developing somatosensory cortex. Nature Communications, 7, 12540. https://doi.org/10.1038/ncomms12540

Mosser, C. A., Baptista, S., Arnoux, I., \& Audinat, E. (2017). Microglia in CNS development: Shaping the brain for the future. Progress in Neurobiology, 149-150, 1-20. https://doi.org/10.1016/j.pneurobio.2017. 01.002

Nimmerjahn, A., Kirchhoff, F., \& Helmchen, F. (2005). Resting microglial cells are highly dynamic surveillants of brain parenchyma in vivo. Science, 308(5726), 1314-1318. https://doi.org/10.1126/science. 1110647

Pagani, F., Paolicelli, R. C., Murana, E., Cortese, B., Di Angelantonio, S., Zurolo, E., ... Ragozzino, D. (2015). Defective microglial development in the hippocampus of $\mathrm{Cx} 3 \mathrm{cr} 1$ deficient mice. Frontiers in Cellular Neuroscience, 9, 111. https://doi.org/10.3389/fncel.2015.00111

Paolicelli, R. C., Bolasco, G., Pagani, F., Maggi, L., Scianni, M., Panzanelli, P., ... Gross, C. T. (2011). Synaptic pruning by microglia is necessary for normal brain development. Science, 333(6048), 1456-1458. https:// doi.org/10.1126/science.1202529

Paolicelli, R. C., \& Gross, C. T. (2011). Microglia in development: Linking brain wiring to brain environment. Neuron Glia Biology, 7(1), 77-83. https://doi.org/10.1017/S1740925X12000105

Parkhurst, C. N., Yang, G., Ninan, I., Savas, J. N., Yates, J. R., 3rd, Lafaille, J. J., ... Gan, W. B. (2013). Microglia promote learning-dependent synapse formation through brain-derived neurotrophic factor. Cell, 155(7), 1596-1609. https://doi.org/10.1016/j.cell. 2013.11.030

Pascual, O., Ben Achour, S., Rostaing, P., Triller, A., \& Bessis, A. (2012). Microglia activation triggers astrocyte-mediated modulation of excitatory neurotransmission. Proceedings of the National Academy of Sciences of the United States of America, 109(4), E197-E205. https://doi. org/10.1073/pnas.1111098109

Pfeiffer, T., Avignone, E., \& Nagerl, U. V. (2016). Induction of hippocampal long-term potentiation increases the morphological dynamics of microglial processes and prolongs their contacts with dendritic spines. Scientific Reports, 6, 32422. https://doi.org/10.1038/srep32422

Piccinin, S., Di Angelantonio, S., Piccioni, A., Volpini, R., Cristalli, G., Fredholm, B. B., ... Ragozzino, D. (2010). CX3CL1-induced modulation at CA1 synapses reveals multiple mechanisms of EPSC modulation involving adenosine receptor subtypes. Journal of Neuroimmunology, 224(1-2), 85-92. https://doi.org/10.1016/j.jneuroim.2010.05.012

Ragozzino, D., Di Angelantonio, S., Trettel, F., Bertollini, C., Maggi, L., Gross, C., ... Eusebi, F. (2006). Chemokine fractalkine/CX3CL1 negatively modulates active glutamatergic synapses in rat hippocampal neurons. The Journal of Neuroscience, 26(41), 10488-10498. https://doi. org/10.1523/JNEUROSCI.3192-06.2006

Renger, J. J., Egles, C., \& Liu, G. (2001). A developmental switch in neurotransmitter flux enhances synaptic efficacy by affecting AMPA receptor activation. Neuron, 29(2), 469-484.

Reshef, R., Kreisel, T., Beroukhim Kay, D., \& Yirmiya, R. (2014). Microglia and their CX3CR1 signaling are involved in hippocampal- but not olfactory bulb-related memory and neurogenesis. Brain, Behavior, and Immunity, 41, 239-250. https://doi.org/10.1016/j.bbi.2014.04.009

Reshef, R., Kudryavitskaya, E., Shani-Narkiss, H., Isaacson, B., Rimmerman, N., Mizrahi, A., \& Yirmiya, R. (2017). The role of microglia and their CX3CR1 signaling in adult neurogenesis in the olfactory bulb. eLife, 6, e30809. https://doi.org/10.7554/eLife.30809

Ricci-Tersenghi, F., Minneci, F., Sola, E., Cherubini, E., \& Maggi, L. (2006). Multivesicular release at developing Schaffer collateral-CA1 synapses: An analytic approach to describe experimental data. Journal of Neurophysiology, 96(1), 15-26. https://doi.org/10.1152/jn.01202.2005

Sallert, M., Rantamaki, T., Vesikansa, A., Anthoni, H., Harju, K., Yli-Kauhaluoma, J., ... Lauri, S. E. (2009). Brain-derived neurotrophic factor controls activity-dependent maturation of CA1 synapses by downregulating tonic activation of presynaptic kainate receptors. The Journal of Neuroscience, 29(36), 11294-11303. https://doi.org/10. 1523/JNEUROSCI.0560-09.2009

Santello, M., Bezzi, P., \& Volterra, A. (2011). TNFalpha controls glutamatergic gliotransmission in the hippocampal dentate gyrus. Neuron, 69(5), 988-1001. https://doi.org/10.1016/j.neuron.2011.02.003

Schafer, D. P., Lehrman, E. K., Kautzman, A. G., Koyama, R., Mardinly, A. R., Yamasaki, R., ... Stevens, B. (2012). Microglia sculpt postnatal neural circuits in an activity and complement-dependent manner. Neuron, 74(4), 691-705. https://doi.org/10.1016/j.neuron.2012.03.026

Schecter, R. W., Maher, E. E., Welsh, C. A., Stevens, B., Erisir, A., \& Bear, M. F. (2017). Experience-dependent synaptic plasticity in V1 occurs without microglial CX3CR1. The Journal of Neuroscience, 37(44), 10541-10553. https://doi.org/10.1523/JNEUROSCI.2679-16.2017

Sellner, S., Paricio-Montesinos, R., Spiess, A., Masuch, A., Erny, D., Harsan, L. A., ... Blank, T. (2016). Microglial CX3CR1 promotes adult neurogenesis by inhibiting Sirt 1/p65 signaling independent of CX3CL1. Acta Neuropathologica Communications, 4(1), 102. https://doi. org/10.1186/s40478-016-0374-8

Semple, B. D., Blomgren, K., Gimlin, K., Ferriero, D. M., \& Noble-Haeusslein, L. J. (2013). Brain development in rodents and humans: Identifying benchmarks of maturation and vulnerability to injury across species. Progress in Neurobiology, 106-107, 1-16. https:// doi.org/10.1016/j.pneurobio.2013.04.001

Singh, P., Hockenberry, A. J., Tiruvadi, V. R., \& Meaney, D. F. (2011). Computational investigation of the changing patterns of subtype specific NMDA receptor activation during physiological glutamatergic neurotransmission. PLoS Computational Biology, 7(6), e1002106. https://doi. org/10.1371/journal.pcbi.1002106

Stevens, C. F., \& Wang, Y. (1994). Changes in reliability of synaptic function as a mechanism for plasticity. Nature, 371(6499), 704-707. https://doi.org/10.1038/371704a0

Tian, L., Hui, C. W., Bisht, K., Tan, Y., Sharma, K., Chen, S., ... Tremblay, M. E. (2017). Microglia under psychosocial stressors along the aging trajectory: Consequences on neuronal circuits, behavior, and brain diseases. Progress in Neuro-Psychopharmacology \& Biological Psychiatry, 79(Pt A), 27-39. https://doi.org/10.1016/j.pnpbp.2017.01.007

Tremblay, M. E., Lowery, R. L., \& Majewska, A. K. (2010). Microglial interactions with synapses are modulated by visual experience. PLoS Biology, 8(11), e1000527. https://doi.org/10.1371/journal.pbio.1000527

Tremblay, M. E., Zettel, M. L., Ison, J. R., Allen, P. D., \& Majewska, A. K. (2012). Effects of aging and sensory loss on glial cells in mouse visual and auditory cortices. Glia, 60(4), 541-558. https://doi.org/10.1002/ glia.22287 
Voronin, L. L., \& Cherubini, E. (2004). Deaf, mute and whispering' silent synapses: Their role in synaptic plasticity. The Journal of Physiology, 557(Pt 1), 3-12. https://doi.org/10.1113/jphysiol.2003.058966

Wake, H., Moorhouse, A. J., Jinno, S., Kohsaka, S., \& Nabekura, J. (2009). Resting microglia directly monitor the functional state of synapses in vivo and determine the fate of ischemic terminals. The Journal of Neuroscience, 29(13), 3974-3980. https://doi.org/10.1523/ JNEUROSCI.4363-08.2009

Wasling, P., Hanse, E., \& Gustafsson, B. (2004). Developmental changes in release properties of the CA3-CA1 glutamate synapse in rat hippocampus. Journal of Neurophysiology, 92(5), 2714-2724. https://doi.org/10. 1152/jn.00464.2004

Weinhard, L., di Bartolomei, G., Bolasco, G., Machado, P., Schieber, N. L., Neniskyte, U., ... Gross, C. T. (2018). Microglia remodel synapses by presynaptic trogocytosis and spine head filopodia induction. Nature Communications, 9(1), 1228. https://doi.org/10.1038/s41467-018-03566-5

Winkler, Z., Kuti, D., Ferenczi, S., Gulyas, K., Polyak, A., \& Kovacs, K. J. (2017). Impaired microglia fractalkine signaling affects stress reaction and coping style in mice. Behavioural Brain Research, 334, 119-128. https://doi.org/10.1016/j.bbr.2017.07.023

Wolf, S. A., Boddeke, H. W., \& Kettenmann, H. (2017). Microglia in physiology and disease. Annual Review of Physiology, 79, 619-643. https:// doi.org/10.1146/annurev-physiol-022516-034406
Yoon, J. Y., \& Choi, S. (2017). Evidence for presynaptically silent synapses in the immature hippocampus. Biochemical and Biophysical Research Communications, 482(4), 1375-1380. https://doi.org/10.1016/j.bbrc. 2016.12.044

Zhan, Y., Paolicelli, R. C., Sforazzini, F., Weinhard, L., Bolasco, G., Pagani, F., ... Gross, C. T. (2014). Deficient neuron-microglia signaling results in impaired functional brain connectivity and social behavior. Nature Neuroscience, 17(3), 400-406. https://doi.org/10.1038/nn. 3641

\section{SUPPORTING INFORMATION}

Additional supporting information may be found online in the Supporting Information section at the end of the article.

How to cite this article: Basilico B, Pagani F, Grimaldi A, et al. Microglia shape presynaptic properties at developing glutamatergic synapses. Glia. 2019;67:53-67. https://doi.org/10. 1002/glia.23508 